\title{
Line-space description of resonant four-wave mixing: Theory for isotropic molecular states
}

\author{
A. Kouzovi, a) and P. Radi ${ }^{2}$ \\ ${ }^{1}$ Department of Physics, Saint-Petersburg State University, Ulyanovskaya str. 3, Peterhof, \\ Saint-Petersburg 198504, Russia \\ ${ }^{2}$ Paul Scherrer Institute, CH-5232 Villigen, Switzerland
}

(Received 26 October 2013; accepted 18 April 2014; published online 15 May 2014)

\begin{abstract}
Based on the quantum Liouville formalism, a theory of the two-color, triply resonant four-wave mixing is developed for molecules with isotropically oriented angular momenta. The approach allows to strictly incorporate the relaxation matrices $\Gamma^{(r)}(r=0,1,2)$ into the third-order susceptibility $\chi^{(3)}$ whose expression acquires therewith the form of a scalar product in the line space. Thanks to this representation, isolation of all resonance terms from $\chi^{(3)}$ becomes a routine task. Some of these terms correspond to the case when a molecule initially interacts with two pump photons of the same frequency. Such interactions give rise to the grating line-space vectors which have the same (zero) eigenfrequency. Due to this degeneracy, the latter are easily mixed by rotationally inelastic collisions which shows up in a state-resolved coherence transfer. The satellite signals induced thereby provide a great scope to study the state-to-state inelastic rates in situ by purely optical means. If the diagonal form of $\Gamma$ is assumed, the satellites become forbidden and our results reduce to conventional expressions for the main resonances. Polarization configurations are designed for direct measurements of the population $(r=0)$, orientation $(r=1)$, and alignment $(r=2)$ contributions to $\chi^{(3)}$. Finally, depending on the photon-molecule interaction sequence, the resonance terms of $\chi^{(3)}$ are shown to be differently affected by velocity averaging, the effect which conspicuously manifests itself when Doppler broadening becomes paramount. (C) 2014 AIP Publishing LLC. [http://dx.doi.org/10.1063/1.4874159]
\end{abstract}

\section{INTRODUCTION}

During the last decades, the Liouville Quantum Mechanics (LQM) has developed into a powerful tool to treat different aspects of molecular dynamics including the cases when the molecular motion is perturbed by interactions with the bath. ${ }^{1}$ Thanks to seminal papers of $\mathrm{Fano}^{2}$ and $\mathrm{Mori}^{3}$ based on the LQM formalism, the relaxation (super)operator $\hat{\Gamma}$ is commonly applied for the theoretical treatment of spectroscopic effects. Under the general name of the Line-Space (LS) approach, it is now widely used to interpret the pressure effects on linear spectroscopic responses ${ }^{4-6}$ from real gases. The diagonal elements of the $\hat{\Gamma}$-matrix are directly accessible to measurements since they are just the collisional line widths of resolved spectra and have the meaning of the decay rates of the combining states. The off-diagonal elements tagged by two pairs of quantum numbers, $(i, k)$ and $(l, m)$, are responsible for the collision-induced transfer between corresponding optical transitions $i \rightarrow k$ and $l \rightarrow m$. Investigations $^{7-9}$ of fundamental properties of $\hat{\Gamma}$ as well as elaboration of its diverse models properly accounting for these properties (see books in Refs. 4 and 5 and the review in Ref. 6) fostered numerous applications to gases of atmospheric and technological importance as well as to studies of collisional relaxation processes themselves. The $\hat{\Gamma}$-matrix is also a key quantity to exhaustively interpret the picosecond laser-induced fluorescence (LIF) responses ${ }^{10}$ and those

\footnotetext{
a)Electronic mail: alex@ak1197.spb.edu
}

obtained by coherent anti-Stokes Raman techniques, both in time $^{11-15}$ and frequency ${ }^{16-18}$ domains. The vibrational band shapes measured by Raman gain/loss techniques ${ }^{19,20}$ should also be acknowledged as sensible tools for checking the $\hat{\Gamma}$ matrix models.

However, none of the aforementioned spectroscopic techniques provide the possibility to directly measure the stateto-state rates which are equal to the negated off-diagonal $\hat{\Gamma}$ matrix elements. The least-square fittings of coalesced band shapes, while enabling one to conclude on the overall quality of a relaxation model, cannot guarantee the accuracy of particular rotational state-to-state rates obtained by fittings. It is also pertinent to note that, except for molecular hydrogen, accurate first-principle calculations of $\hat{\Gamma}$ are still unfeasible, primarily because a huge number of matrix elements is needed for bandshape simulations.

Because of the energy exchange between photons and molecules, incoherent responses are produced in the conventional (linear) spectroscopic devices. On the contrary, such an exchange can be forbidden when signals are generated by nonlinear methods. The simplest nonlinear process in an isotropic medium requires three photons to address a molecule in such a way that a fourth photon is simultaneously created in a hitherto empty field mode. This scheme is implemented in a four-wave mixing setup operating with three incident beams, two of the same frequency $\Omega_{1}=\Omega_{2}$ and the third of a different frequency $\Omega_{3}$, all crossed in a small volume from which a fourth beam at $\Omega_{4}=\Omega_{3}$ emerges. This spatially resolved signal becomes enormously enhanced when $\Omega_{1}, \Omega_{2}$, 
and $\Omega_{3}$ approach molecular self-frequencies. Molecules thus act as catalyst boosting a resonant redistribution of photons between field modes. This spectroscopic method is referred to as Two-Color Resonant Four-Wave Mixing (2C-RFWM). The simpler one-color (1C) RFWM version for which three input beams (with $\Omega_{1}=\Omega_{2}=\Omega_{3}=\Omega_{4}$ ) are produced by the same laser is appreciated as an efficient mean for hot gas diagnostics in combustion studies. Different 1C- and 2CRFWM-based techniques were realized (see books in Refs. 21 and 22) now forming a rapidly expanding branch of optical spectroscopy.

2C-RFWM advantageously stands out against all other spectroscopic tools, for its fingerprints can be directly associated with particular state-to-state collisional transfer characteristics. More specifically, the (quasi) continuous-wave $2 \mathrm{C}-\mathrm{RFWM}^{23}$ provides a way to measure cross-correlation times $^{24,25}$ in real gases while an even richer picture of the state-to-state transfer is accessible by picosecond 2C-RFWM pump-probe techniques. ${ }^{26-28}$ As shown below, the LS approach provides the most efficient means to develop a general 2C-RFWM theory for isotropic systems, i.e., for those with completely disoriented molecular angular momenta. The combination of the LS formalism with the Irreducible Spherical Tensor (IST) algebra ${ }^{29}$ extensively accounts for the symmetry of the problem. As demonstrated by Williams et al. ${ }^{30-32}$ the IST recoupling techniques offer the easiest way to cast the resultant $2 \mathrm{C}$-RFWM amplitudes into products of the field and molecular characteristics.

Contrary to the direct multiple summations over magnetic quantum numbers as used before, ${ }^{30-32}$ the present procedure is divided into step-by-step calculations of few LS matrix elements. Advantageously, the LS vectors are symmetry-adapted yielding matrix elements that need much less algebraic work and make the whole calculation less prone to errors. Of even greater importance is that the LS approach allows a rigorous incorporation of the exact relaxation matrix into the third-order susceptibility $\left(\chi^{(3)}\right)$ expression, much in common with the procedure used for linear spectroscopies. ${ }^{1}$ Based on this result, one can separate the resonance terms corresponding to conventional responses ${ }^{30-32}$ as well as to weaker satellites induced by the state-to-state transfer. The frequency-domain theory of these effects is extensively expounded in this paper with details omitted in our previous publications. $^{24,25}$ In doing so, all possible 2C-RFWM excitation schemes, including those leading to the satellite induction, are scrutinized.

Thus, one of the goals pursued by this study is to advertise a tandem of the LS and IST techniques as a novel efficient tool to interpret collisional signatures in nonlinear optical responses from isotropic media. The tandem techniques detailed and extensively used in this study are prerequisite to extend the 2C-RFWM theory to more complicated systems and, as a groundwork, seem necessary for a concise presentation of new results in forthcoming publications.

Among the first candidate systems are molecular ensembles with anisotropic angular momentum and velocity distributions. The first investigation ${ }^{33}$ of the laser-pumped states of the $\mathrm{CS}_{2}$ molecule by degenerate (or one-color, 1C) RFWM revealed the high potential of the method as a new, purely optical tool to study rotational anisotropy. It should be emphasized that the RFWM polarization setup, as compared to that of LIF, possesses two additional degrees of freedom and therefore can be tailored to extract finer characteristics of the angular momentum distribution which remain beyond the reach of other spectroscopic techniques. Very recently, ${ }^{34}$ 1C-RFWM was successfully employed to study anisotropic features of the nascent $\mathrm{OH}$ radicals produced by laser photolysis of hydrogen peroxide. The results hold much promise for investigations of reaction pathways and further stress the need to advance an adequate theoretical picture.

The paper is organized as following. We first outline the basics of the LS approach and then derive with its help a general expression of $\chi^{(3)}$. Next, we switch to a detailed analysis of the triply resonant terms contributing to both the main and satellite 2C-RFWM responses. The polarization dependence is considered and new effects are predicted for the signal dependences when different combinations of the pumped and probed states are used. Also, the velocity averaging needed to account for the Doppler effect on the RFWM line intensities is considered in a more detailed way than done in previous publications. These results are crucial to quantitatively interpret the main and satellite RFWM responses from $\mathrm{OH}$ and NH-containing flames which will be treated in a separate publication. In this study, we thus concentrate on the key problems encountered in the analysis of the resonance part of $\chi^{(3)}\left(\Omega_{4} ; \Omega_{3}, \Omega_{2}, \Omega_{1}\right)$. Once it is completed, the calculation of the integrated RFWM intensities becomes a routine task. The shape calculations requiring multiple convolutions of $\left|\chi^{(3)}\right|^{2}$ with the laser intensity distributions can be done as in the CARS case ${ }^{35}$ these calculations, however, exceed the scope of this study.

\section{LIOUVILLE APPROACH}

With the help of LQM, the scalar form ${ }^{36}$ of $\chi^{(3)}\left(\Omega_{4}\right.$; $\Omega_{3}, \Omega_{2}, \Omega_{1}$ ) in the frequency domain is compactly written as $^{22}$

$$
\begin{aligned}
\chi^{(3)}\left(\Omega_{4} ; \Omega_{3}, \Omega_{2}, \Omega_{1}\right)= & N \sum_{P} \operatorname{Tr} \rho_{T} \hat{V}_{a}\left(\Omega_{a}^{\prime}-\hat{L}+i 0\right)^{-1} \hat{V}_{b} \\
& \times\left(\Omega_{a}^{\prime}+\Omega_{b}^{\prime}-\hat{L}+i 0\right)^{-1} \hat{V}_{c} \\
& \times\left(\Omega_{a}^{\prime}+\Omega_{b}^{\prime}+\Omega_{c}^{\prime}-\hat{L}+i 0\right)^{-1} d_{4},
\end{aligned}
$$

where $\rho_{T}$ and $\hat{L}=\hat{L}_{S}+\hat{L}_{B}+\hat{L}_{1}$ are, respectively, the density matrix and the total Liouvillian of the "molecule(S)+bath(B)" system; the coupling between both subsystems is represented by the term $\hat{L}_{1}$. A small imaginary constant $i \varepsilon(\varepsilon>0)$ is to be subtracted from $\hat{L}$ in order to fit the condition ${ }^{6}$ that the interaction between the electromagnetic field and the $\mathrm{S}+\mathrm{B}$ system started in the infinitely remote past $(t \rightarrow-\infty)$; finally, the limit $\varepsilon \rightarrow 0$ should be taken in (1) that is symbolically denoted by $i 0$. From Eq. (1) on, the Liouvillian (super)operators are hatted to distinguish them from the ordinary (unhatted) quantum operators. The common factor $N=n_{0} / 6 \hbar^{3}$ scales linearly 
with the molecular number density $n_{0} ; d_{k}=\left(\mathbf{e}_{k}, \mathbf{d}\right)$ is the projection of the molecular dipole moment $\mathbf{d}$ onto the unit polarization vector $\mathbf{e}_{k}$ of the absorbed photon $k(k=1,3)$. For the emitted photons $(k=2,4)$, the complex conjugate vector $\mathbf{e}_{k}^{*}$ is to be used, i.e., $d_{k}=\left(\mathbf{e}_{k}^{*}, \mathbf{d}\right)$. Evidently, the conjugation affects the results only when elliptically (or circularly) polarized beams are used. As commonly assumed, the frequency $\Omega_{2}$ in Eq. (1) should be negated while the positive frequencies $\Omega_{1}$ and $\Omega_{3}$ are ascribed to the absorbed photons. The field-molecule dipole coupling Liouvillian $\hat{V}_{k}$ acts on an arbitrary operator $X$ as a commutator: $\hat{V}_{k} X \equiv-\left[d_{k}, X\right]$. The symbol $\sum_{P}$ denotes the summation over all possible $3 !=6$ permutations of frequencies $\Omega_{a}, \Omega_{b}, \Omega_{c}$ of three incident waves, their polarizations $\mathbf{e}_{j}$ and wave vectors $\mathbf{k}_{j}(j$ $=a, b, c)$. The field energy conservation, i.e., $\hbar \Omega_{4}=\hbar\left(\Omega_{a}\right.$ $\left.+\Omega_{b}+\Omega_{c}\right)=\hbar\left(\Omega_{1}-\Omega_{2}+\Omega_{3}\right)$, is a prerequisite for the coherent 4-wave mixing generation. Due to macroscopic averaging over the molecular positions, the generated beam propagates in the direction of $\mathbf{k}_{4}=\mathbf{k}_{1}-\mathbf{k}_{2}+\mathbf{k}_{3}$ (the phase conjugation condition). In Eq. (1), the Doppler-shifted frequencies are primed, $\Omega_{j}^{\prime}=\Omega_{j}-\left(\mathbf{k}_{j}, \mathbf{v}\right)$, where $\mathbf{v}$ is the molecular velocity. Totally, $3 ! 2^{3}=48$ terms contribute to (1).

The bath averaging routine ${ }^{24}$ (see Appendix A) converts (1) into

$$
\chi^{(3)}\left(\Omega_{4}^{\prime} ; \Omega_{3}^{\prime}, \Omega_{2}^{\prime}, \Omega_{1}^{\prime}\right) \approx \operatorname{Tr} \tilde{\rho}_{S} \hat{T} d_{4}\left[1+O\left(n_{B}\right)\right] .
$$

Here, $n_{B}$ stands for the number density of the bath particles and $\hat{T}$ is obtained from the Liouvillian superoperator acting on $d_{4}$ in Eq. (1) in which $\hat{L}$ has to be substituted by $\hat{L}_{S}-i \hat{\Gamma}$. In the LS theory, ${ }^{1}$ the relaxation operator $\hat{\Gamma}$ can be further divided into the dynamical $\left(\hat{\Gamma}^{\prime}\right)$ and the static $(\Delta \hat{\Omega})$ terms (see Appendix A). Since we consider here only gases at moderate or low pressures, all terms of $\chi^{(3)}$ in (2) which vary linearly (or faster) with $n_{B}$ will be neglected.

The molecular density matrix $\tilde{\rho}_{S}$ in Eq. (2) is defined as an average of $\rho_{T}$ over the bath states and, since the bath is assumed to be isotropic, so is $\tilde{\rho}_{S}$. This implies $\tilde{\rho}_{S}$ to be completely diagonal in the magnetic quantum numbers $(M)$ associated with the total molecular angular momentum j. Following the commonly adopted procedure, $\tilde{\rho}_{S}$ will be approximated by the density matrix $\rho_{S}$ of a free molecule. A semiclassical form of the latter matrix will be used, $\rho_{S}=\rho M(\mathbf{v})$, where the diagonal matrix $\rho$ is associated with rovibronic degrees of freedom and $M(\mathbf{v})$ is the classical Maxwellian velocity distribution.

Consider operators $A, B, C, \ldots$ acting on the molecular rovibronic eigenfunctions $|\alpha J M\rangle$ where $\alpha$ is a shorthand notation of the rovibronic principal quantum numbers except $J$. The line space $\mathcal{L}_{S}$ is defined as a manifold of these operators with the metric

$$
\langle\langle A \mid B\rangle\rangle \equiv \operatorname{Tr} \tilde{\rho}_{S} A^{\dagger} B,
$$

where $A^{\dagger}$ is the hermitian conjugate of $A$. When $A\left(=A_{q_{a}}^{\left(r_{a}\right)}\right)$ and $B\left(=B_{q_{b}}^{\left(r_{b}\right)}\right)$ are ISTs of ranks $r_{a}$ and $r_{b}$, their scalar product survives only when $r_{a}=r_{b}$ and $q_{a}=q_{b}$ and is independent on the projection label $q$. Due to this, we shall omit sometimes the projection labels (when this does not create ambiguity). Because of the ensemble isotropy, $\mathcal{L}_{S}$ is splitted into subspaces $\mathcal{L}_{S}^{(r)}(r=0,1,2 \ldots): \mathcal{L}_{S}=\mathcal{L}_{S}^{(0)} \oplus \mathcal{L}_{S}^{(1)}$ $\oplus \mathcal{L}_{S}^{(2)} \oplus \cdots$ corresponding to the irreducible representations of the group $O(3)$. The basis in $\mathcal{L}_{S}^{(r)}$ is composed of the symmetry-adapted $m \rightarrow n$ transition vectors ${ }^{7}$

$$
\begin{aligned}
\left.\left|K_{\sigma}^{(r)}\right\rangle\right\rangle= & \rho_{m m}^{-1 / 2} \sum_{M_{n} M_{m}}(-1)^{J_{m}-M_{m}} C_{J_{n} M_{n} J_{m}-M_{m}}^{r \sigma}\left|\alpha_{n} J_{n} M_{n}\right\rangle \\
& \times\left\langle\alpha_{m} J_{m} M_{m}|\equiv|\left(m n^{\times}\right)_{\sigma}^{(r)}\right\rangle \mid,
\end{aligned}
$$

where $C_{J_{n} M_{n} J_{m}-M_{m}}^{r \sigma}$ are the Clebsch-Gordan coefficients and the ket component is labeled by a tilted cross. For brevity, the sets of the principal quantum numbers will be hereafter labeled by a single letter, $i=\left(\alpha_{i}, J_{i}\right)$. It is easy to verify that the vectors (4) are normalized and orthogonal in respect to the metric (3)

$$
\left\langle\left\langle K_{1 \sigma_{1}}^{\left(r_{1}\right)} \mid K_{2 \sigma_{2}}^{\left(r_{2}\right)}\right\rangle\right\rangle=\delta_{m_{1} m_{2}} \delta_{n_{1} n_{2}} \delta_{r_{1} r_{2}} \delta_{\sigma_{1} \sigma_{2}}
$$

In this basis, $\hat{L}_{S}$ is completely diagonal with eigenvalues $\omega_{n m}$ $=\left(E_{n}-E_{m}\right) / \hbar \equiv \omega_{K}$ whereas the relaxation matrix $\hat{\Gamma}$ is blockshaped and couples the LS vectors only with the same parity change $p^{7}$

$$
\left\langle\left\langle K_{1 \sigma_{1}}^{\left(r_{1}\right)}|\Gamma| K_{2 \sigma_{2}}^{\left(r_{2}\right)}\right\rangle\right\rangle=\Gamma_{K_{1} K_{2}}\left(r_{1}\right) \delta_{r_{1} r_{2}} \delta_{\sigma_{1} \sigma_{2}} \delta_{p_{1} p_{2}} .
$$

Here, a shorthand LS notation of the $\Gamma$-matrix element was used; when the clarity reasons demand, all four quantum states involved will be supplied, i.e., $\Gamma_{K_{1} K_{2}}\left(r_{1}\right)=\Gamma_{n_{1} m_{1}}^{n_{2} m_{2}}\left(r_{1}\right)$. Using (6), the leading term of (2) can be written as a matrix element of the product of six LS operators (cf Eqs. (1) and (2))

$$
\begin{aligned}
\chi^{(3)}= & \left\langle\left\langle I|T| d_{4}\right\rangle\right\rangle \\
= & N \sum_{P} \sum\left\langle\left\langle I\left|V_{a}\right| K_{1 \sigma_{1}}^{\left(r_{1}\right)}\right\rangle\right\rangle\left\langle\left\langle K_{1 \sigma_{1}}^{\left(r_{1}\right)}\left|R_{r_{1}}\left(\Omega_{a}^{\prime}\right)\right| K_{1^{\prime} \sigma_{1}}^{\left(r_{1}\right)}\right\rangle\right\rangle \\
& \times\left\langle\left\langle K_{1^{\prime} \sigma_{1}}^{\left(r_{1}\right)}\left|V_{b}\right| K_{2 \sigma_{2}}^{\left(r_{2}\right)}\right\rangle\right\rangle\left\langle\left\langle K_{2 \sigma_{2}}^{\left(r_{2}\right)}\left|R_{r_{2}}\left(\Omega_{a}^{\prime}+\Omega_{b}^{\prime}\right)\right| K_{2^{\prime} \sigma_{2}}^{\left(r_{2}\right)}\right\rangle\right\rangle \\
& \times\left\langle\left\langle K_{2^{\prime} \sigma_{2}}^{\left(r_{2}\right)}\left|V_{c}\right| K_{3 \sigma_{3}}^{\left(r_{3}\right)}\right\rangle\right\rangle\left\langle\left\langle K_{3 \sigma_{3}}^{\left(r_{3}\right)}\right| R_{r_{3}}\left(\Omega_{a}^{\prime}+\Omega_{b}^{\prime}+\Omega_{c}^{\prime}\right)\right. \\
& \left.\times\left|K_{3^{\prime} \sigma_{3}}^{\left(r_{3}\right)}\right\rangle\right\rangle\left\langle\left\langle K_{3^{\prime} \sigma_{3}}^{\left(r_{3}\right)} \mid d_{4}\right\rangle\right\rangle,
\end{aligned}
$$

where $I=\sum_{i}|i\rangle\langle i|$ is the identity operator, $\hat{R}_{r}\left(\Omega^{\prime}\right)=\left(\Omega^{\prime}-\right.$ $\left.\hat{L}_{S}+i 0+i \hat{\Gamma}(r)\right)^{-1}$ are the resolvent (super)operators and the summations under the sign of $\sum$ should be performed over all intermediate LS vectors, their ranks $r_{k}$ and the projections $\sigma_{k}(k=1,2,3)$. Since in respect to molecular rotations $\hat{V}_{k}$ and $d_{k}$ are ISTs of rank one, the ranks $r_{1}$ and $r_{3}$ should also equal unity so that $K_{1 \sigma_{1}}^{\left(r_{1}\right)}$ and $K_{3 \sigma_{3}}^{\left(r_{3}\right)}$ correspond to the dipoleallowed transitions. The LS matrix element of $V_{b}$ in (7) obeys the vector addition rule $\mathbf{r}_{1}+\mathbf{1}=\mathbf{r}_{2}$; hence, $r_{2}$ may be 0,1 , or 2. Due to Eq. (6), the resolvent matrix elements depend only on the ranks $r_{k}$ which facilitates the summations over $\sigma_{1}, \sigma_{2}$, and $\sigma_{3}$ in (7). The reader is referred to Appendix B where the derivation of the required LS matrix elements is outlined. Advantageously, the routine leads to decoupling of the molecular and field characteristics. 
The resultant general expression for $\chi^{(3)}$ fully accounting for the collisional relaxation reads

$$
\begin{aligned}
\chi^{(3)}= & N \sum_{P} \sum_{r=0,1,2} \sum_{n_{i} m_{i} n_{i}^{\prime} m_{i}^{\prime}}\left(\rho_{m_{1} m_{1}}-\rho_{n_{1} n_{1}}\right) d_{m_{1} n_{1}} d_{n_{3}^{\prime} m_{3}^{\prime}}(-1)^{J_{n_{1}}-J_{m_{1}}+J_{n_{1}^{\prime}}-J_{m_{1}^{\prime}}+J_{n_{2}^{\prime}}-J_{m_{2}^{\prime}}} \\
& \times \sqrt{\frac{\rho_{m_{1}^{\prime} m_{1}^{\prime}} \rho_{m_{2}^{\prime} m_{2}^{\prime}} \rho_{m_{3} m_{3}}}{\rho_{m_{1} m_{1}} \rho_{m_{2} m_{2}} \rho_{m_{3}^{\prime} m_{3}^{\prime}}}}\left\langle\left\langle\left(m_{1} n_{1}^{\times}\right)^{(1)}\left|R_{1}\left(\Omega_{a}^{\prime}\right)\right|\left(m_{1}^{\prime} n_{1}^{\prime \times}\right)^{(1)}\right\rangle\right\rangle \\
& \times\left\langle\left\langle\left(m_{2} n_{2}^{\times}\right)^{(r)}\left|R_{r}\left(\Omega_{a}^{\prime}+\Omega_{b}^{\prime}\right)\right|\left(m_{2}^{\prime} n_{2}^{\prime \times}\right)^{(r)}\right\rangle\right\rangle\left\langle\left\langle\left(m_{3} n_{3}^{\times}\right)^{(1)}\left|R_{1}\left(\Omega_{4}^{\prime}\right)\right|\left(m_{3}^{\prime} n_{3}^{\prime \times}\right)^{(1)}\right\rangle\right\rangle \\
& \times\left\{\delta_{m_{1}^{\prime} m_{2}} d_{n_{1}^{\prime} n_{2}} T_{n_{2} n_{1}^{\prime} m_{2}}^{r}\left[\delta_{m_{2}^{\prime} m_{3}} d_{n_{2}^{\prime} n_{3}} T_{n_{2}^{\prime} n_{3} m_{2}^{\prime}}^{r} G_{a b c 4}(r)-\delta_{n_{2}^{\prime} n_{3}} T_{n_{2}^{\prime} m_{3} m_{2}^{\prime}}^{r} d_{m_{3} m_{2}^{\prime}} G_{a b 4 c}(r)\right]\right. \\
& \left.+\delta_{n_{1}^{\prime} n_{2}} d_{m_{2} m_{1}^{\prime}} T_{n_{2} m_{1}^{\prime} m_{2}}^{r}\left[\delta_{n_{2}^{\prime} n_{3}} d_{m_{3} m_{2}^{\prime}} T_{n_{2}^{\prime} m_{3} m_{2}^{\prime}}^{r} G_{b a 4 c}(r)-\delta_{m_{2}^{\prime} m_{3}} d_{n_{2}^{\prime} n_{3}} T_{n_{2}^{\prime} n_{3} m_{2}^{\prime}}^{r} G_{b a c 4}(r)\right]\right\},
\end{aligned}
$$

where $d_{m n}=\langle m\|d\| n\rangle$ are the reduced matrix elements of the dipole moment and $T_{k l m}^{r} \equiv\left\{\begin{array}{ccc}1 & r & 1 \\ J_{k} & J_{l} & J_{m}\end{array}\right\}$ are $6 \mathrm{j}$-symbols. The field characteristics, or the polarization factors,

$$
G_{i j k l}(r)=\left(\left\{e_{i}^{(1)} \otimes e_{j}^{(1)}\right\}^{(r)},\left\{e_{k}^{(1)} \otimes e_{l}^{(1)}\right\}^{(r)}\right)
$$

are scalar quantities build up on $\mathbf{e}_{n}$ treated as ISTs of rank one, $e_{n}^{(1)}(n=1,2,3,4)$; here, contraction ${ }^{29}$ of two ISTs, $A^{(a)}$ and $B^{(b)}$, into an IST of rank $c$ was denoted by $\left\{A^{(a)} \otimes B^{(b)}\right\}^{(c)}$. Conventionally, the $r$-dependent contributions are called population $(r=0)$, orientation $(r=1)$, and alignment $(r=2)$. Also, the LS vectors $\left.\left|\left(m n^{\times}\right)^{(r)}\right\rangle\right\rangle$ (4) may be classified under two types, Raman (for which $n \neq m$ and the eigenfrequencies $\omega_{n m}$ are nonzero) and grating ${ }^{32}$ (those with $m=n$ which refer to the zero eigenfrequency).

To separate the resonance part, $\chi_{\text {res }}^{(3)}$, we have to identify the virtual states in the derived spectral expansion (8) with those which are resonantly pumped and probed. In doing so, we shall assume the off-diagonal elements of $\hat{\Gamma}$ to be much smaller than the splittings between the lines. In this case, lines do not interfere and the Diagonal Approximation (DA) for the matrix elements of resolvents in (8) holds

$$
\begin{aligned}
& \left\langle\left\langle\left(m n^{\times}\right)^{(r)}\left|R_{r}\left(\Omega^{\prime}\right)\right|\left(m^{\prime} n^{\prime \times}\right)^{(r)}\right\rangle\right\rangle \\
& \quad \approx \delta_{m m^{\prime}} \delta_{n n^{\prime}} /\left(\Omega^{\prime}-\omega_{n m}+i \Gamma_{n m}^{n m}(r)\right) \\
& \equiv \delta_{m m^{\prime}} \delta_{n n^{\prime}} L_{r}\left(\Omega^{\prime}-\omega_{n m}+i \Gamma_{n m}^{n m}(r)\right) .
\end{aligned}
$$

Hence, we have $m_{1}^{\prime}=m_{1}, n_{1}=n_{1}^{\prime}, m_{3}^{\prime}=m_{3}$, and $n_{3}^{\prime}=n_{3}$. Evidently, all resolvents are automatically diagonalized in the off-resonance cases (when the detunings much exceed the $\Gamma$ matrix elements) which reduces Eq. (8) to the much simpler conventional expression ${ }^{37}$ of $\chi^{(3)}$.

The DA disregards the collisional intensity transfer between closely spaced lines, the so-called line mixing effect, ${ }^{5}$ which increasingly manifests itself with the growing line overlap. For this reason, the DA is inapplicable for grating transitions since they occur at the same (zero) frequency and hence are completely overlapping. We notice first that in the grating subspaces the $\hat{L}_{S}$-matrix disappears. Thus, if the eigenspectrum of $\hat{\Gamma}(r)$ contains a zero eigenvalue, the on-resonance resolvent $R_{r_{2}}(0)=[i \varepsilon+i \hat{\Gamma}(r)]^{-1}$ becomes singular in the $\varepsilon=0$ limit. As shown in Ref. 8 , the LS vectors which solely depend on coordinates are annulled by $\hat{\Gamma}$, $\left.\hat{\Gamma}(r)\left|A^{(r)}\right\rangle\right\rangle=0$, which implies that they correspond to the zero eigenvalue. However, in the orientation and alignment cases one cannot build up a grating vector which is diagonal in the coordinate representation, for the expansion of such $\left.\left|A^{(r)}\right\rangle\right\rangle$ $(r=1,2)$ in the LS basis (4) always contains the Raman terms that moves the vector out from the grating subspace. Besides, the $m \neq n$ transitions are far detuned from the zero frequency and thus negligibly affect the grating resolvents. Therefore, if the matrix operations are performed within the $r=1$ and $r=2$ grating subspaces, the $R_{1}\left(\Omega^{\prime}\right)$ and $R_{2}\left(\Omega^{\prime}\right)$ can be treated as nonsingular matrices. With the additional proviso that the off-diagonal terms of $\hat{\Gamma}(r)(r=1,2)$ are much smaller than the diagonal terms, one can apply (10) at $m$ $=n$ as a reasonable approximation, though much less accurate and controllable than for spectroscopically resolved lines.

The inversion problem is aggravated in the $r=0$ case since the identity operator $I_{S}=\sum_{m}|m\rangle\langle m| \in \mathcal{L}_{S}^{(0)}$ is annulled $^{8}$ by $\hat{\Gamma}(0)$ and thus can be considered as its eigenvector, $\left.\left|A_{0}^{(0)}\right\rangle\right\rangle$, with the eigenvalue $\gamma_{0}(0)=0$. However, we should first perform the inversion at finite $\varepsilon$ that is easily accomplished once all eigenvectors $\left.\left|A_{k}^{(0)}\right\rangle\right\rangle(k=0,1,2, \ldots)$ and eigenvalues $\gamma_{k}(0)$ are known

$$
\begin{aligned}
\left.R_{0}\left(\Omega^{\prime}\right)\left|K_{2^{\prime}}^{(0)}\right\rangle\right\rangle= & \left.\sum_{k=0,1,2 \ldots}\left[\Omega^{\prime}+i \varepsilon+i \gamma_{k}(0)\right]^{-1}\left|A_{k}^{(0)}\right\rangle\right\rangle \\
& \times\left\langle\left\langle A_{k}^{(0)} \mid K_{2^{\prime}}^{(0)}\right\rangle\right\rangle
\end{aligned}
$$

where the projection labels have been dropped for brevity, and then take the limit $\varepsilon \rightarrow 0$. Omitting for a moment the identity operator, $\left.\sum_{K_{2}}\left|K_{2}^{(0)}\right\rangle\right\rangle\left\langle\left\langle K_{2}^{(0}\right|\right.$, which was introduced to (7) just to explicitly show the LS spectral expansion structure, we see that the operation (11) is to be followed by the action of $\hat{V}_{b}$. The latter annuls $\left.\left.\left|A_{0}^{(0)}\right\rangle\right\rangle=\left|I_{S}\right\rangle\right\rangle$, for such vector obviously commutes with $d_{b}$. We thus arrive at

$$
\begin{aligned}
& \left.\lim _{\varepsilon \rightarrow 0} \hat{V}_{b} R_{0}\left(\Omega^{\prime}\right)\left|K_{2^{\prime}}^{(0)}\right\rangle\right\rangle \\
& \left.\quad=\hat{V}_{b} \sum_{k=1,2, \ldots}\left[\Omega^{\prime}+i \gamma_{k}(0)\right]^{-1}\left|A_{k}^{(0)}\right\rangle\right\rangle\left\langle\left\langle A_{k}^{(0)} \mid K_{2^{\prime}}^{(0)}\right\rangle\right\rangle .
\end{aligned}
$$


By expanding $\left.\left|A_{k}^{(0)}\right\rangle\right\rangle$ in the LS basis, we obtain

$$
\begin{aligned}
& \left\langle\left\langle K_{2}^{(0)}\left|R_{0}\left(\Omega^{\prime}\right)\right| K_{2^{\prime}}^{(0)}\right\rangle\right\rangle \\
& \quad=\sum_{k=1,2, \ldots}\left[\Omega^{\prime}+i \gamma_{k}(0)\right]^{-1}\left\langle\left\langle K_{2}^{(0)} \mid A_{k}^{(0)}\right\rangle\right\rangle\left\langle\left\langle A_{k}^{(0)} \mid K_{2^{\prime}}^{(0)}\right\rangle\right\rangle
\end{aligned}
$$

that merely means that at $\varepsilon=0$ the inversion should be performed in the subspace of $\mathcal{L}_{S}^{(0)}$ which is orthogonal to $\left.\left.\left|A_{0}^{(0)}\right\rangle\right\rangle=\left|I_{S}\right\rangle\right\rangle$. To calculate the main resonances of $\chi^{(3)}$, the diagonal elements with $K_{2}^{(0)}=K_{2^{\prime}}^{(0)}$ are required. In the light of the preceding, the approximation $\left\langle\left\langle K_{2}^{(0)}\left|R_{0}(0)\right| K_{2}^{(0)}\right\rangle\right\rangle$ $\approx 1 / i \Gamma_{K_{2} K_{2}}$ postulated in Refs. 30-32 can be hardly justified.

The resonance conditions along with the DA set obvious limitations on the molecular states in Eq. (8). First, $\Omega_{4}$ is supposedly tuned to $\omega_{n_{3} m_{3}}$ that fixes the quantum numbers of the upper $\left(n_{3}\right)$ and lower $\left(m_{3}\right)$ states between which the generation occurs. Besides, the frequency $\Omega_{a}$ of the first attacker should be close either to $\omega_{n_{1} m_{1}}\left(\right.$ when $\Omega_{a}>0$ ) or to $\omega_{m_{1} n_{1}}$ (when $\Omega_{a}<0$ ) whereas the states $n_{2}$ and $m_{2}$ should satisfy the equation $\Omega_{a}+\Omega_{b}=\omega_{n_{2} m_{2}}$. Hence, gratings can be formed only when $\Omega_{a}+\Omega_{b}=0$. Finally, the compatibility of the terms I-IV of Eq. (8) proportional to the Kronecker deltas $\delta_{m_{1}^{\prime} m_{2}} \delta_{m_{2}^{\prime} m_{3}}(\mathrm{I}), \quad \delta_{m_{1}^{\prime} m_{2}} \delta_{n_{2}^{\prime} n_{3}}(\mathrm{II}), \quad \delta_{n_{1}^{\prime} n_{2}} \delta_{n_{2}^{\prime} n_{3}}$ (III), and $\delta_{n_{1}^{\prime} n_{2}} \delta_{m_{2}^{\prime} m_{3}}$ (IV) with the above restrictions should be verified for the six possible interaction sequences, 123, 213, 231, 321, 312 , and 132, corresponding to permutations in Eqs. (1), (7), and (8). This procedure completely specifies the quantum numbers of the resonating virtual states and thus resolves all allowed partial contributions to $\chi_{\text {res }}^{(3)}$. The thus derived $\chi_{\text {res }}^{(3)}$ expression may however vary depending on a particular excitation scheme (see Sec. III).

\section{THREE-LEVEL SCHEMES}

Consider first the conventional 3-level schemes (Fig. 1) which are responsible for generation of the main RFWM responses. For these schemes, the pumped and probed states have a common level, either in the ground (UP scheme) or in the excited (SEP and UNFOLDED) state. For definiteness, lasers 1 and 2 will be further assumed to pump the $i \rightarrow f$ transition.
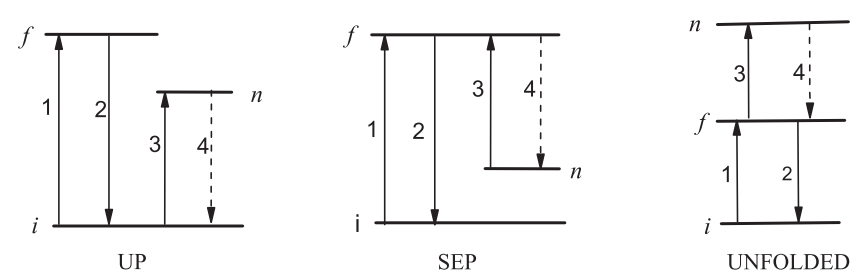

FIG. 1. Three-level schemes used to generate TC-RFWM by pumping transitions from the ground state $i ; 1$ and 2 label pump beams, 3 and 4 label, respectively, the probe and signal beams. Arrows pointing upwards denote absorption of a photon, those pointing downwards correspond to emission. There are two additional SEP and UNFOLDED schemes obtained by interchanging the beam labels: $1 \leftrightarrows 3$ and $2 \leftrightarrows 4$. In these cases, transitions from the excited $n$ (SEP scheme) and $f$ (UNFOLDED scheme) levels are pumped.

\section{A. SEP scheme}

As a guiding example, the SEP scheme (Fig. 1) will be closely examined in the DA; other schemes can be analyzed similarly and less details will be given. In the SEP configuration, the state $f$ assumedly belongs to the excited electronic level whereas the states $n$ and $i$ are in the ground electronic manifold and are the only populated ones. In the considered case, the $n \rightarrow f$ transition is probed by laser 3 so that $n_{3}=f, m_{3}=n$ always holds. To start with, we consider contributions generated by term I whose proportionality to $\delta_{m_{1} m_{2}} \delta_{m_{2}^{\prime} m_{3}}$ fixes $m_{1}=m_{2}, m_{2}^{\prime}=m_{3}=n$. For this term, gratings may arise provided $m_{2}^{\prime}=n_{2}^{\prime}=n, m_{2}=n_{2}=m_{1}$. Besides, their formation is possible only when the first two attacking photons have the same energy and opposite frequencies, $\Omega_{a}+\Omega_{b}=0$. Therefore, the interaction sequences causing the grating effect are always (123) and (213). In the (123) case, the resonance condition, $\Omega_{1}=\omega_{f i}$, demands that $m_{1}$ $=i, n_{1}=f$. The thus derived relations, $m_{2}=i=n_{2}$ and $m_{2}^{\prime}=n_{2}^{\prime}=n$, imply that sequence (123) generates the intensity transfer $i \rightarrow n$ in the ground-state grating. By similar arguments we obtain for sequence (213) that $m_{1}=m_{2}=n_{2}$ $=f, n_{1}=i, m_{2}^{\prime}=n_{2}^{\prime}=m_{3}=n$ which means that the grating is formed in the upper electronic state and that it contributes to the 2C-RFWM amplitude via the collisional $f \rightarrow n$ transfer to the ground electronic state. Such a contribution can be safely neglected because the (assumed) large energy gap results in an infinitesimal transfer probability. Next, we can conclude that sequences (132) and (312) are inactive since the frequency $\Omega_{1}+\Omega_{3}$ is out of resonance. The Raman-type contribution originating from sequence (231) demands $\Omega_{3}-\Omega_{2}$ to be in resonance with $\omega_{i n}$, i.e., $n_{2}=i, m_{2}=n$ which contradicts to the resonance condition of $\Omega_{2}=\omega_{f i}$ from which we have $n_{1}=i, m_{1}=f=m_{2}$. Thus, the $\mathrm{I}(231)$ contribution is forbidden. However, the photon sequence (321) is contributing with $m_{1}=m_{2}=m_{2}^{\prime}=m_{3}=n, n_{1}=f=n_{3}, n_{2}^{\prime}$ $=n_{2}=i$. Totally, term I contributes to $\chi_{S E P}^{(3)}$ via

$$
\begin{aligned}
\chi_{S E P}^{(3)}(I)= & C_{S E P} \sum_{r}\left[\rho_{n n}(-1)^{J_{i}-J_{n}+r} G_{1423}(r)\right. \\
& \times L_{1}\left(\Omega_{3}^{\prime}-z_{f n}\right) L_{r}\left(\Omega_{3}^{\prime}-\Omega_{2}^{\prime}-z_{i n}\right) T_{i f n}^{r} T_{i f n}^{r} \\
& +G_{1234}(r) \sqrt{\rho_{n n} \rho_{i i}} L_{1}\left(\Omega_{1}^{\prime}-z_{f i}\right) \\
& \left.\times\left\langle\left\langle\left(i i^{\times}\right)^{(r)}\left|R\left(\Omega_{1}^{\prime}-\Omega_{2}^{\prime}\right)\right|\left(n n^{\times}\right)^{(r)}\right\rangle\right\rangle T_{i f i}^{r} T_{n f n}^{r}\right],
\end{aligned}
$$

where $z_{l m}=\omega_{l m}-i \Gamma_{l m}^{l m}(r)$ and

$$
C_{S E P}=N\left|d_{f i} d_{n f}\right|^{2} L_{1}\left(\Omega_{4}^{\prime}-z_{f n}\right) \text {. }
$$

From Eq. (14) on, to present the results in a more symmetric form, the coupling order of ISTs forming the $G$-factors will be sometimes changed by using formulas of Ref. 29.

As Eq. (14) shows, the allowed (Raman) and collisioninduced terms become negligibly small as the population factor $\rho_{n n}$ tends to zero. However, even in the case of a sufficiently populated level $n$, the collision-induced term can be wiped out by a small transfer probability as it holds for large gaps $\left|\hbar \omega_{n i}\right|$. In most practical cases, this term is negligible when $n$ and $i$ states belong to different vibronic levels. 
Term II (with $m_{1}=m_{2}, n_{2}^{\prime}=n_{3}$ ) is compatible only with the 213 order and produces the upper-state grating contribution at $m_{1}=m_{2}=n_{2}=m_{2}^{\prime}=n_{2}^{\prime}=n_{3}=f, n_{1}=i, m_{3}=n$

$$
\begin{aligned}
\chi_{S E P}^{(3)}(I I)= & C_{S E P} \rho_{i i} L_{1}\left(-\Omega_{2}^{\prime}-z_{i f}\right) \sum_{r} G_{1234}(r) \\
& \times\left\langle\left\langle\left(f f^{\times}\right)^{(r)}\left|R\left(\Omega_{1}^{\prime}-\Omega_{2}^{\prime}\right)\right|\left(f f^{\times}\right)^{(r)}\right\rangle\right\rangle T_{f i f}^{r} T_{f n f}^{r} .
\end{aligned}
$$

The only contribution from term III $\left(n_{1}=n_{2}, n_{2}^{\prime}=n_{3}\right)$ occurs via the 123 sequence which creates the $f$-state grating with $m_{1}=i, n_{3}=n_{2}=n_{2}^{\prime}=m_{2}=m_{2}^{\prime}=n_{1}=f, m_{3}=n$

$$
\begin{aligned}
\chi_{S E P}^{(3)}(I I I)= & C_{S E P} \rho_{i i} L_{1}\left(\Omega_{1}^{\prime}-z_{f i}\right) \sum_{r} G_{1234}^{(r)} \\
& \times\left\langle\left\langle\left(f f^{\times}\right)^{(r)}\left|R\left(\Omega_{1}^{\prime}-\Omega_{2}^{\prime}\right)\right|\left(f f^{\times}\right)^{(r)}\right\rangle\right\rangle T_{f i f}^{r} T_{f n f}^{r} .
\end{aligned}
$$

Finally, term IV $\left(n_{1}=n_{2}, m_{2}^{\prime}=m_{3}\right)$ produces the grating 213 (with $m_{1}=n_{3}=f, n_{1}=n_{2}=m_{2}=i, n_{2}^{\prime}=m_{2}^{\prime}=m_{3}=n$ ) and Raman 231 (with $m_{1}=n_{3}=f, n_{1}=n_{2}=n_{2}^{\prime}=i, m_{2}$ $\left.=m_{2}^{\prime}=m_{3}=n\right)$ amplitudes

$$
\begin{aligned}
& \chi_{S E P}^{(3)}(I V) \\
& =C_{S E P} \rho_{i i} L_{1}\left(-\Omega_{2}^{\prime}-z_{i f}\right) \\
& \quad \times \sum_{r}\left[G_{1234}(r) \sqrt{\frac{\rho_{n n}}{\rho_{i i}}}\left\langle\left\langle\left(i i^{\times}\right)^{(r)}\left|R\left(\Omega_{1}^{\prime}-\Omega_{2}^{\prime}\right)\right|\left(n n^{\times}\right)^{(r)}\right\rangle\right\rangle\right. \\
& \quad \times T_{i f i}^{r} T_{n f n}^{r} \\
& \left.\quad+(-1)^{J_{i}-J_{n}+r} G_{1423}(r) L_{r}\left(\Omega_{3}^{\prime}-\Omega_{2}^{\prime}-z_{i n}\right) T_{i f n}^{r} T_{i f n}^{r}\right] . \text { (18) }
\end{aligned}
$$

The derived results show how the net SEP amplitude is built up of six terms. Four of them comprise the main (allowed) contribution to $\chi_{S E P}^{(3)}$ and do not require the collisional intensity transfer. Three terms out of four correspond to the situation when the molecule starts from the (pumped) level $i$ whereas for the fourth term it starts from the probed level $n$. While the first three allowed contributions have been derived $^{32}$ using the diagrammatic techniques, the remaining one was unaccounted for. It appears to be quite important whenever the populations $\rho_{n n}$ and $\rho_{i i}$ are comparable. For instance, when the levels $n$ and $i$ approach each other, as is in the 1C-RFWM case, the total amplitude consists of 8 terms, four stemming from the SEP expression. Without a proper account for the term proportional to $\rho_{n n}$, the SEP-type contribution to the total 1C-RFWM amplitude would be incomplete. Interestingly, in the limit $\rho_{n n} / \rho_{i i} \rightarrow \infty$ our result reproduces the situation treated by Williams et al. (scheme $1 \mathrm{~b}$ of Ref. 32) in which the lower pumped level was assumedly unpopulated. It should be noted that the three allowed amplitudes derived in the foregoing are in a full accordance with the previously obtained results. ${ }^{32}$ All allowed contributions can be depicted by conventional double-sided Feynman diagrams. ${ }^{37}$ Besides, our theory predicts two additional terms caused by the $i \rightarrow n$ intensity transfer in the ground-state $(i)$ grating. It is worth noting that the inverse $n \rightarrow i$ transfer does not contribute since the gratings can be formed only in the pumped

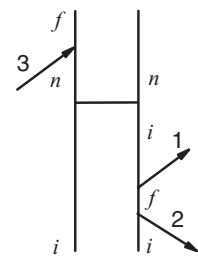

(a)

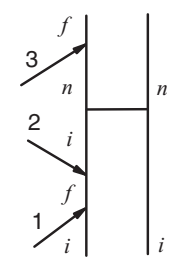

(b)

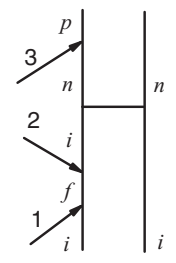

(c)

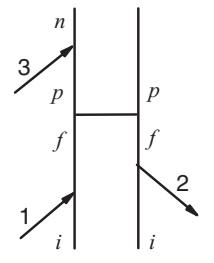

(d)
FIG. 2. 2C-RFWM double-sided Feynman diagrams accounting for inelastic collisional effects. Horizontal line represents collisional coupling of grating states. Panels (a) and (b): Contributions to the SEP amplitude due to transfer in the ground-state grating Panels (c) and (d): Collision-induced amplitudes produced by interaction sequence 123 and caused by transfer in the (c) ground- and (d) upper-state gratings. Diagram (d) refers to the extended UNFOLDED scheme on Fig. 3(b).

states but not in the probed one $(n)$. The transfer-induced contributions are proportional to the on-resonance off-diagonal matrix element of the relevant resolvent and relate to the cross correlation time $\tau_{\text {in }}$ between the two grating vectors, $\left(i i^{\times}\right)^{(r)}$ and $\left(n n^{\times}\right)^{(r)}$

$$
\begin{aligned}
\tau_{i n}(r) & \equiv i^{-1} \lim _{\Delta \Omega \rightarrow 0}\left\langle\left\langle\left(i i^{\times}\right)^{(r)}|R(\Delta \Omega)|\left(n n^{\times}\right)^{(r)}\right\rangle\right\rangle \\
& =\int_{0}^{\infty}\left\langle\left\langle\left(i i^{\times}\right)^{(r)}\left|e^{-\Gamma(r) t}\right|\left(n n^{\times}\right)^{(r)}\right\rangle\right\rangle d t .
\end{aligned}
$$

Likewise, the diagonal $(f-f)$ matrix element contributing to terms II (16) and III (17) has the meaning of the collisional correlation time $\tau_{f f}^{(r)}$ of the $f$-state grating of rank $r$. The collisional intensity transfer can be straightforwardly incorporated into conventional diagrams depicting the 2C-RFWM amplitudes. ${ }^{32,37}$ For that, the relaxation should be represented by a horizontal segment connecting the vertical bra and ket evolution lines as exemplified in Fig. 2. The graphs on panels (a) and (b) (Fig. 2) depict two collision-induced terms of the SEP amplitude appearing on the right-hand sides of Eqs. (14) and (18).

\section{B. UP scheme}

In the UP case, the level $n$ assumedly belongs to the same electronic state as the upper pumped level $f$, both having negligibly small populations; however, the ratio $\rho_{n n} / \rho_{f f}$ is perceived to be finite. The terms I-IV contribute to the total amplitude via the following sequences: (I) 123 , (II) 231, (II) 213 , (III) 321, and (IV) 213. The net UP amplitude is equal to

$$
\begin{aligned}
\chi_{U P}^{(3)}= & C_{U P} \rho_{i i} \sum_{r}\left\{G_{1234}^{(r)}\left[L_{1}\left(\Omega_{1}^{\prime}-z_{f i}\right)+L_{1}\left(-\Omega_{2}^{\prime}-z_{i f}\right)\right]\right. \\
& \times\left[\left\langle\left\langle\left(i i^{\times}\right)^{(r)}\left|R\left(\Omega_{1}^{\prime}-\Omega_{2}^{\prime}\right)\right|\left(i i^{\times}\right)^{(r)}\right\rangle\right\rangle T_{i f i}^{r} T_{i n i}^{r}\right. \\
& \left.+\sqrt{\frac{\rho_{n n}}{\rho_{f f}}}\left\langle\left\langle\left(f f^{\times}\right)^{(r)}\left|R\left(\Omega_{1}^{\prime}-\Omega_{2}^{\prime}\right)\right|\left(n n^{\times}\right)^{(r)}\right\rangle\right\rangle T_{f i f}^{r} T_{n i n}^{r}\right] \\
& +(-1)^{J_{n}-J_{f}+r} G_{1423}(r) L_{r}\left(\Omega_{3}^{\prime}-\Omega_{2}^{\prime}-z_{n f}\right) T_{n i f}^{r} T_{n i f}^{r} \\
& \left.\times\left[L_{1}\left(\Omega_{3}^{\prime}-z_{n i}\right)+L_{1}\left(-\Omega_{2}^{\prime}-z_{i f}\right)\right]\right\},
\end{aligned}
$$


where

$$
C_{U P}=N\left|d_{f i} d_{n i}\right|^{2} L_{1}\left(\Omega_{4}^{\prime}-z_{n i}\right) .
$$

The term (II) 213 is responsible for the grating formation in the $f$-state from which the coherence is transfered to the $n$ state by collisional relaxation. The $i \rightarrow n$ transfer was disregarded in (20) because these levels assumedly belong to different vibronic states.

\section{UNFOLDED scheme}

Denote the ground, medium, and upper levels by $i, f$, and $n$ (Fig. 1); again, only the ground state $i$ is assumedly populated. Besides, we suppose that the gaps between these states are large enough to annul the interstate collisional transfer. The contributing terms, I(213), II(132), and IV(123), produce the amplitude

$$
\begin{aligned}
\chi_{U N F}^{(3)}= & -C_{U N F} \rho_{i i} \sum_{r}(-1)^{r}\left\{G _ { 1 2 3 4 } ( r ) \left[L_{1}\left(-\Omega_{2}^{\prime}-z_{i f}\right)\right.\right. \\
& \left.+L_{1}\left(\Omega_{1}^{\prime}-z_{f i}\right)\right] T_{f i f}^{r} T_{f n f}^{r} \\
& \times\left\langle\left\langle\left(f f^{\times}\right)^{(r)}\left|R\left(\Omega_{1}^{\prime}-\Omega_{2}^{\prime}\right)\right|\left(f f^{\times}\right)^{(r)}\right\rangle\right\rangle \\
& +(-1)^{J_{n}-J_{i}} G_{1324}(r) T_{n f i}^{r} T_{n f i}^{r} L_{1}\left(\Omega_{1}^{\prime}-z_{f i}\right) \\
& \left.\times L_{r}\left(\Omega_{1}^{\prime}+\Omega_{3}^{\prime}-z_{n i}\right)\right\},
\end{aligned}
$$

where

$$
C_{U N F}=N\left|d_{f i} d_{f n}\right|^{2} L_{1}\left(\Omega_{4}^{\prime}-z_{n f}\right) .
$$

Finally, a modified UNFOLDED scheme (Fig. 1) should also be considered for which the pump lasers 1 and 2 are tuned to the $f \rightarrow n$ transition and the $i \rightarrow f$ transition is probed by laser 3. In this case, only the I(312) term survives for which $m_{1}=m_{2}=m_{3}=i, n_{1}=n_{3}=f$, and $n_{2}=n$.

\section{COLLISION-INDUCED SATELLITE RESONANCES}

They appear in the general four-level scheme because of intensity transfer in both the ground (i)- and upper $(f)$-state gratings. Panel (a) of Fig. 3 depicts extended SEP and UP schemes for which the levels $n$ and $i$ assumedly belong to the ground vibronic state while the states $p$ and $f$ are in the excited vibronic manifold. The satellite resonance at $\omega_{p n}$ is sourced by the $i \rightarrow n$ and $f \rightarrow p$ collisional transfers. There

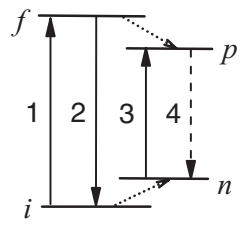

(a)

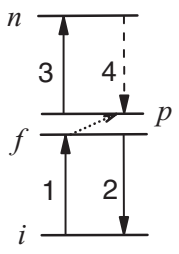

(b)
FIG. 3. (a) and (b) Four-level scheme. Satellites are generated either because of the collisional $i \rightarrow n$ transfer in the ground state grating or because of the $f \rightarrow p$ transfer in the upper state grating. are four contributions, I(123), II(213), III(123), and IV(213), to the collision-induced amplitude whose expression reads

$$
\begin{aligned}
& \chi_{I N D}^{(3)}(S E P / U P) \\
& =C_{I N D} \rho_{i i}\left[L_{1}\left(\Omega_{1}^{\prime}-z_{f i}\right)+L_{1}\left(-\Omega_{2}^{\prime}-z_{i f}\right)\right] \\
& \quad \times \sum_{r} G_{1234}(r)\left[\sqrt{\frac{\rho_{n n}}{\rho_{i i}}}\right. \\
& \quad \times\left\langle\left\langle\left(i i^{\times}\right)^{(r)}\left|R\left(\Omega_{1}^{\prime}-\Omega_{2}^{\prime}\right)\right|\left(n n^{\times}\right)^{(r)}\right\rangle\right\rangle T_{i f i}^{r} T_{n p n}^{r}+\sqrt{\frac{\rho_{p p}}{\rho_{f f}}} \\
& \left.\times\left\langle\left\langle\left(f f^{\times}\right)^{(r)}\left|R\left(\Omega_{1}^{\prime}-\Omega_{2}^{\prime}\right)\right|\left(p p^{\times}\right)^{(r)}\right\rangle\right\rangle T_{f i f}^{r} T_{p n p}^{r}\right] \\
& C_{I N D}=N\left|d_{i f} d_{n p}\right|^{2} L_{1}\left(\Omega_{4}^{\prime}-z_{p n}\right) .
\end{aligned}
$$

One of the induction channels, say $f \rightarrow p$, can be closed if the $p$ level belongs to a vibronic manifold different from that of the state $f$. Equation (24) extends our previous results $^{24,25}$ derived by perturbation theory. In the modified SEP scheme (when the pump lasers operate between unpopulated states), no grating is formed and, therefore, its four-level extension cannot lead to satellite generation. When both $\rho_{n n}$ and $\rho_{i i}$ are distinct from zero the modified SEP scheme produces gratings in both pumped states and the resulting collision-induced amplitudes can be obtained from Eq. (24) by an obvious renumeration of levels. With the proviso that $\rho_{f f}$ and $\rho_{p p}$ are negligibly small (but its ratio is finite), the UNFOLDED scheme (b) of Fig. 3 gives rise just to two contributions, I(213) and IV(123), induced by the $f \rightarrow p$ transfer in the $f$-state grating

$$
\begin{aligned}
\chi_{I N D}^{(3)}(U N F) \\
=-C_{I N D} \rho_{i i} \sum_{r}(-1)^{r} G_{1234}(r) \\
\quad \times\left[L_{1}\left(\Omega_{1}^{\prime}-z_{f i}\right)+L_{1}\left(-\Omega_{2}^{\prime}-z_{i f}\right)\right] \sqrt{\frac{\rho_{p p}}{\rho_{f f}}} \\
\quad \times\left\langle\left\langle\left(f f^{\times}\right)^{(r)}\left|R\left(\Omega_{1}^{\prime}-\Omega_{2}^{\prime}\right)\right|\left(n n^{\times}\right)^{(r)}\right\rangle\right\rangle T_{f i f}^{(r)} T_{p n p}^{(r)} .
\end{aligned}
$$

One of two diagrams referring to this case is drawn on panel (d) of Fig. 2; it depicts the induction due to transfer in the $f$-state gratings formed by the 123 interaction sequence. The modified UNFOLDED scheme (see comments in the caption of Fig. 1) cannot produce satellites, for the only contributing sequence, 312 , does not create a grating.

Under the binary collision regime, the relaxation rates entering $\chi_{\text {res }}^{(3)}$ scale linearly with the gas density $n_{0}$ so that the peak values of both the allowed and satellite intensities vary in the same fashion (as $n_{0}^{-4}$ ) and thus their ratio is invariant with pressure. These ratios are thus thermal-averaged, pressureindependent characteristics of a binary collision. As a rough guide, the allowed intensities are proportional to the square of the inverse total rate for a molecule to leave the combining (pumped) states whereas the satellite intensity is determined by the inverse rate of a particular radiationless transition from these states. The intensity ratio of such resonances characterizes a relative contribution of the particular transition rate to 
the total decay rate. More reliable results on the relative rates can be obtained by considering the ratios of integrated intensities. This, in turn, requires averaging over the velocities of the emitters (as discussed in Sec. VII) which can affect the weights characterizing contributions of particular interaction sequences to $\chi_{r e s}^{(3)}$.

\section{POLARIZATION DEPENDENCE}

The $G(r)$-factors defined by (9) are known ${ }^{29}$

$$
\begin{gathered}
G_{a b c d}(0)=\left(\mathbf{e}_{a}, \mathbf{e}_{b}\right)\left(\mathbf{e}_{c}, \mathbf{e}_{d}\right) / 3, \\
G_{a b c d}(1)=-\left[\left(\mathbf{e}_{a}, \mathbf{e}_{c}\right)\left(\mathbf{e}_{b}, \mathbf{e}_{d}\right)-\left(\mathbf{e}_{a}, \mathbf{e}_{d}\right)\left(\mathbf{e}_{b}, \mathbf{e}_{c}\right)\right] / 2, \\
G_{a b c d}(2)=\left(\mathbf{e}_{a}, \mathbf{e}_{c}\right)\left(\mathbf{e}_{b}, \mathbf{e}_{d}\right) / 2-\left(\mathbf{e}_{a}, \mathbf{e}_{b}\right)\left(\mathbf{e}_{c}, \mathbf{e}_{d}\right) / 3 \\
+\left(\mathbf{e}_{a}, \mathbf{e}_{d}\right)\left(\mathbf{e}_{b}, \mathbf{e}_{c}\right) / 2,
\end{gathered}
$$

that considerably facilitates the analysis. For three-level schemes, the grating terms are proportional to $G_{1234}(r)$ whereas the Raman-type resonances contain $G_{1423}(r)$ (SEP and UP schemes) or $G_{1324}(r)$ (UNFOLDED scheme). Since the satellites emerge because of the grating formation they depend only on the $G_{1234}$-factors. The presence of polarization factors with different photon orderings much complicates the analysis of the allowed resonances. However, when the $r$-dependence of the resolvents is negligible the Raman and grating SEP/UNFOLDED terms can be transformed via

$$
\sum_{r}(-1)^{r+J_{i}-J_{n}} G_{1423}(r) T_{n f i}^{r} T_{n f i}^{r}=\sum_{r} G_{1234}(r) T_{f i f}^{r} T_{f n f}^{r},
$$

$$
\sum_{r}(-1)^{J_{n}-J_{i}+r} G_{1324}(r) T_{n f i}^{r} T_{n f i}^{r}=\sum_{r} G_{2134}(r) T_{f i f}^{r} T_{f n f}^{r},
$$

which generalizes the results of Bloch and Ducloy ${ }^{38}$ for the degenerate RFWM case. To transform the sum of the Raman UP terms (see (20)), Eq. (30) can be applied with interchanged labels $i$ and $f$. Equations (30) and (31) are proved in Appendix $\mathrm{C}$ to explicate the techniques which will be later used by us for anisotropic states. Actually, Eqs. (30) and (31) illustrate the invariance of the 2C-RFWM transition matrix elements to the order of summations over magnetic quantum numbers. ${ }^{30,32}$ By using these equations, the SEP/UP and UNFOLDED amplitudes can be expressed via the $G_{a b c d}(r)$ coefficients with a fixed photon ordering (say, 1234). Remarkably, the time-domain 2C-RFWM techniques permit to control the interaction time orderings which substantially simplifies the analysis of signals. For instance, only two sequences, 1234 and 2134, may contribute to a signal generated in the picosecond pump-probe $2 \mathrm{C}$-RFWM setup ${ }^{27}$ and in view of the commutativity of polarization vectors the response becomes determined solely by the $G_{1234}(r)$ factors. The dependence of the $\Gamma$-matrix elements on $r$ stems from the dephasing (elastic) contributions to the rotational broadening at $r=1,2$. Numerical data on these contributions are rather scarce. For room-temperature molecular hydrogen ${ }^{39}$ and nitrogen, ${ }^{9,40}$ they amount up to $20 \%$. The fittings ${ }^{25}$ of the $2 \mathrm{C}$ RFWM spectra of $\mathrm{OH}$ in an atmospheric flame with the fast collision relaxation model ${ }^{9}$ showed considerably smaller part (about $5 \%$ ) of rotational dephasing, in conformity with the recent time-domain measurements of the 2C-RFWM spectra of $\mathrm{OH}^{27}$ This trend agrees with the collision theory predictions about an increase of the inelastic and a simultaneous reduction of the elastic contributions as the temperature is raised. Besides, quenching in the excited electronic states can add to the decay rates and, hence, further reduces their $r$-dependence. Therefore, rearrangement of terms containing such rates based on Eqs. (30) and (31) is less liable to errors than the same operation with the terms proportional to the ground-state rates. The thus derived intensity expressions of the main responses will be used in a subsequent publication as a reference to calibrate satellite signals. Upon such rearrangements, information on the $r$ dependent effects in the ground electronic state can be partly obtained. For that, we need to find the polarization configurations $\left(\mathbf{e}_{1}, \mathbf{e}_{2}, \mathbf{e}_{3}, \mathbf{e}_{4}\right)$ for which only one $r$-term contributes. As a showcase, we assume the transformations (30) and (31) to be applied to eliminate all $G_{a b c d}(r)$-factors except those with $\mathbf{e}_{a}=\mathbf{e}_{1}, \mathbf{e}_{b}=\mathbf{e}_{2}^{*}, \mathbf{e}_{c}=\mathbf{e}_{3}, \mathbf{e}_{d}=\mathbf{e}_{4}^{*}$. In the light of the preceding, this procedure should be used for the UP and UNFOLDED schemes whereas the SEP amplitude should be rather given via the $G_{1423}(r)$ coefficients. Most commonly, plane-polarized co-propagating beams are used with mutually perpendicular or parallel polarization vectors. ${ }^{30,32}$ For such angles, the population, orientation, and alignment terms remain mixed and cannot be separately measured. However, their separation can be designed using tilted geometries. For instance, when $\mathbf{e}_{1} \| \mathbf{e}_{2}$ and $\mathbf{e}_{3} \| \mathbf{e}_{4}$ we have $G_{1234}(0)$ $=1 / 3, G_{1234}(1)=0, G_{1234}(2)=\left(\mathbf{e}_{1}, \mathbf{e}_{3}\right)^{2}-1 / 3$, and, hence, to annul the alignment term, we should simultaneously rotate $\mathbf{e}_{3}$ and $\mathbf{e}_{4}$ by the magic angle $\varphi_{m}=54.7^{\circ}$ for which $\left(\mathbf{e}_{1}, \mathbf{e}_{3}\right)^{2}=1 / 3$. At the $\left(X, Y, X^{\prime}, X^{\prime}\right)$ geometry, the signal from isotropic media is completely washed out when $O X^{\prime} \| O X$ or $O X^{\prime} \| O Y$. At other angles $\varphi$ between $O X^{\prime}$ and $O X, G_{1234}(0)$ and $G_{1234}(1)$ are annulled whereas $G_{1234}(2)=\cos \varphi \sin \varphi$ and its maximal value $(1 / 2)$ is reached at $\varphi=\pi / 4$. Next, in the $(X$, $\left.Y, X^{\prime}, Y^{\prime}\right)$ case with $O Y^{\prime} \perp O X^{\prime}$ we obtain $G_{1234}(0)=0, G_{1234}(1)$ $=1 / 2$, and $G_{1234}(2)=\cos (2 \varphi) / 2$. Therefore, by choosing $\varphi$ $=\pi / 4$, we can separate the orientation contribution. Similar analysis can be performed in the case of the left $(L)$ and right $(R)$ circular polarizations. When applying Eqs. (27)-(29), we should use the equalities $\left(\mathbf{e}_{R}, \mathbf{e}_{R}^{*}\right)=\left(\mathbf{e}_{L}, \mathbf{e}_{L}^{*}\right)=1,\left(\mathbf{e}_{L}, \mathbf{e}_{R}^{*}\right)$ $=-\left(\mathbf{e}_{L}, \mathbf{e}_{L}\right)=\left(\mathbf{e}_{R}, \mathbf{e}_{L}^{*}\right)=-\left(\mathbf{e}_{R}, \mathbf{e}_{R}\right)=0$. However, having in the disposal circularly polarized beams only, one cannot design configurations in which a single $r$-contribution survives. For that, combinations of circular and plane polarizations are needed. For instance, at the $(R, R, X, Y)$ geometry we have $G_{1234}(0)=0, G_{1234}(1)=-i, G_{1234}(2)=0$. Other geometries at which the orientation contribution becomes separable are $(R, R, Y, X)$ (proposed in Ref. 27), $(L, L, X, Y)$ and $(L, L$, $Y, X)$. The $(R, L, X, X)$, combination, as well as the $(L, R$, $X, X),(R, L, Y, Y)$, and $(L, R, X, X)$ ones, separates the alignment term since $G_{1234}(0)=G_{1234}(1)=0, G_{1234}(2)=-1$. However, we could not find any combination of the circular and plane polarizations allowing to directly measure the 
population contribution. The use of elliptically polarized beams can possibly solve this problem but seems to be impractical. Thus, the plane polarization setup furnishes an optimal mean to directly deduce information on all tensor characteristics of RFWM. We finally notice that the interchangings $\left(\mathbf{e}_{1} \rightleftarrows \mathbf{e}_{2}^{*}, \mathbf{e}_{3} \rightleftarrows \mathbf{e}_{4}^{*}\right), \quad\left(\mathbf{e}_{1} \rightleftarrows \mathbf{e}_{3}, \mathbf{e}_{2}^{*} \rightleftarrows \mathbf{e}_{4}^{*}\right)$, and $\left(\mathbf{e}_{1} \rightleftarrows \mathbf{e}_{4}^{*}, \mathbf{e}_{2}^{*}\right.$ $\left.\rightleftarrows \mathbf{e}_{3}\right)$ do not affect the $G_{1234}(r)$ factors and result therefore in a number of equivalent polarization geometries. From the above it appears that the SEP amplitude (Eqs. (14)-(18)) should be preferably expressed via the $G_{1423}(r)$ factors which can be analyzed in the same way as $G_{1234}(r)$.

\section{QUASICLASSICAL GEOMETRICAL FACTORS}

When the dependence of the relaxation parameters on $r$ is negligible, the RFWM intensities can be expressed via the so-called geometrical factors $G_{F}{ }^{32}$ which are functions of the polarization geometry and the angular momentum quantum numbers. Precisely, in the SEP case these factors are defined via

$$
G_{F}^{S E P}\left(J_{i}, J_{f}, J_{n}\right)=\left(2 J_{f}+1\right) \sum_{r=0,1,2} G_{1234}(r) T_{f i f}^{r} T_{f n f}^{r}
$$

whereas $G_{F}^{U P}$ is obtained by interchanging $J_{i}$ and $J_{f}$ in the right-hand side of (32); $G_{F}^{U N F}$ is also given by (32) but with the interchanged labels 1 and 2 of the pump photons. The $G_{F}$-factors have been analytically calculated for particular setups consisting either of mutually perpendicular or parallel polarizations ${ }^{30,31,41}$ or of left and right circularly polarized $\mathbf{e}_{k}(k=1,2,3,4) .{ }^{30,31}$ Switching from one configuration to another redistributes the intensities in the $2 \mathrm{C}$ - or $1 \mathrm{C}-\mathrm{RFWM}$ branches but never leads to their suppression.

Later, a nonrectangular 1C-RFWM polarization setup was designed by Bracamonte and Vaccaro ${ }^{42}$ who assumed $\mathbf{e}_{1}=\mathbf{e}_{3}$ to be directed along $\mathbf{e}_{Y}$ while the directions of $\mathbf{e}_{2}$ and $\mathbf{e}_{4}$ were varied. The derived formulas showed that particular choices of the $\mathbf{e}_{2}$ and $\mathbf{e}_{4}$ directions could strongly dampen the intensities of different rotational branches. Specifically, by using $\mathbf{e}_{X}$ as a reference axis with $\phi=0$ and by setting $\phi_{2}=45^{\circ}$, elimination of the Q-lines (i.e., those for which $\Delta J=J_{f}-$ $J_{i}=0$ ) or of the P-/R-lines (with $\Delta J=\mp 1$ ) was achieved at high $J_{\mathrm{S}}$ by simply switching the detected direction from $\phi_{4}$ $=-18.43^{\circ}$ to $\phi_{4}=26.57^{\circ}$. Such dissections are very useful to interpret the congested spectra typical to heavy rotators. Here, we shall analyze a more complicated, 2C-RFWM case with the only confinement imposed by the high- $J$ limit.

First, we notice that such values of $J$ favor the introduction of the $G_{F}$-factors (32), since $\Gamma$ depends on $r$ solely because of its elastic, or $\mathbf{j}$-reorientation, terms. The latter decrease with growing $J$ which is interpreted as stabilization of a faster rotating microscopic gyroscope to an action of outer perturbations. Moreover, the elastic contributions are known to fade away with increasing $J$ somewhat faster than the inelastic ones. ${ }^{9,40}$ Besides, high temperatures further shift the balance in favor of the inelastic components of $\Gamma$ as indeed was found in our preceding study of the $\mathrm{OH} 2 \mathrm{C}-\mathrm{RFWM}$ spectra in a flame. ${ }^{25}$
TABLE I. Weights of $G_{1234}(r)$ in geometrical factors (32) at large $J_{\mathrm{s}}$; the upper and lower signs in the $r=1$ row refer to UP/SEP and UNFOLDED schemes, respectively.

\begin{tabular}{ccccc}
\hline \hline$r$ & $R R, P P$ & $P R, R P$ & $Q R, Q P, R Q, R P$ & $Q Q$ \\
\hline 0 & $1 / 3$ & $1 / 3$ & $-1 / 3$ & $1 / 3$ \\
1 & $\pm 1 / 6$ & $\mp 1 / 6$ & 0 & 0 \\
2 & $1 / 30$ & $1 / 30$ & $1 / 15$ & $2 / 15$ \\
\hline \hline
\end{tabular}

In the quasiclassical $(J \gg 1)$ limit, the $6 j$-symbols in (32) reduce to the Clebsch-Gordan coefficients, ${ }^{29}$

$$
T_{J^{\prime} J J^{\prime}}^{r} \approx-\frac{1}{\sqrt{2 J^{\prime}(2 r+1)}} C_{1-\Delta J 1 \Delta J}^{r 0}
$$

provided the difference $\Delta J=J^{\prime}-J$ is much smaller than $J^{\prime}$ and $J$. Asymptotically, the weights of $G_{1234}(r)$ in (32) become $J$-independent and are collected in Table I.

Using their values and Eqs. (27)-(29), we obtain the asymptotic $G_{F}^{U P}=G_{F}^{S E P}$ geometrical factors:

$$
\begin{aligned}
& G_{F}(P P / R R)= \frac{1}{30}\left[3\left(\mathbf{e}_{1}, \mathbf{e}_{2}^{*}\right)\left(\mathbf{e}_{3}, \mathbf{e}_{4}^{*}\right)-2\left(\mathbf{e}_{1}, \mathbf{e}_{3}\right)\left(\mathbf{e}_{2}^{*}, \mathbf{e}_{4}^{*}\right)\right. \\
&\left.+3\left(\mathbf{e}_{1}, \mathbf{e}_{4}^{*}\right)\left(\mathbf{e}_{2}^{*}, \mathbf{e}_{3}\right)\right] \\
& G_{F}(P R / R P)= \frac{1}{30}\left[3\left(\mathbf{e}_{1}, \mathbf{e}_{2}^{*}\right)\left(\mathbf{e}_{3}, \mathbf{e}_{4}^{*}\right)+3\left(\mathbf{e}_{1}, \mathbf{e}_{3}\right)\left(\mathbf{e}_{2}^{*}, \mathbf{e}_{4}^{*}\right)\right. \\
&\left.-2\left(\mathbf{e}_{1}, \mathbf{e}_{4}^{*}\right)\left(\mathbf{e}_{2}^{*}, \mathbf{e}_{3}\right)\right] \\
& G_{F}(Q R / R Q / Q P / P Q) 1 \\
&=\frac{1}{30}\left[-4\left(\mathbf{e}_{1}, \mathbf{e}_{2}^{*}\right)\left(\mathbf{e}_{3}, \mathbf{e}_{4}^{*}\right)\right. \\
&\left.+\left(\mathbf{e}_{1}, \mathbf{e}_{3}\right)\left(\mathbf{e}_{2}^{*}, \mathbf{e}_{4}^{*}\right)+\left(\mathbf{e}_{1}, \mathbf{e}_{4}^{*}\right)\left(\mathbf{e}_{2}^{*}, \mathbf{e}_{3}\right)\right] \\
&\left.\quad+\left(\mathbf{e}_{1}, \mathbf{e}_{4}^{*}\right)\left(\mathbf{e}_{2}^{*}, \mathbf{e}_{3}\right)\right]
\end{aligned}
$$

Equations (36) and (37) also hold for the UNFOLDED case; for other branch combinations, one should use the equalities $G_{F}^{U N F}(P P / R R)=G_{F}^{U P}(P R / R P)$ and $G_{F}^{U N F}(P R / R P)$ $=G_{F}^{U P}(P P / R R)$. From these formulas, the asymptotic values of $G_{F}$ for parallel/perpendicular polarizations from Refs. 30 and 32 are readily reproduced. Interestingly, geometries with plane-polarized beams can be designed to annul any one of the factors (34)-(37). For instance, if we want to dampen the $P P$ - and $R R$-intensities in the SEP and UP spectra, we set $\mathbf{e}_{1}=\mathbf{e}_{3}, \mathbf{e}_{2}=\mathbf{e}_{4}$ and direct $\mathbf{e}_{1}$ at the magic angle to $\mathbf{e}_{2}$. Similarly, in the $P R / R P$ cases, one should set $\mathbf{e}_{1}=\mathbf{e}_{4}, \mathbf{e}_{2}=\mathbf{e}_{3}$ and direct $\mathbf{e}_{2}$ at $\varphi_{m}$ to $\mathbf{e}_{1} ;$ at this geometry, all $\mathrm{QP} / \mathrm{QR} / \mathrm{PQ} / \mathrm{RQ}$ signals are also suppressed. In the $1 \mathrm{C}-\mathrm{RFWM}$ case, the derived results also hold for the $P P-, R R-$, and $Q Q-$ pump/probe combinations; the branch dissection recipes of Ref. 42 also follow from Eqs. (34) and (37). Finally, to suppress the $Q Q$ responses, we direct $\mathbf{e}_{1} \perp \mathbf{e}_{2}, \mathbf{e}_{3} \perp \mathbf{e}_{4}$ and the angle between $\mathbf{e}_{1}$ and $\mathbf{e}_{3}$ should equal $45^{\circ}$. We remind that these simple results are due to the neglect of elastic contributions to the decay rates. Hence, if the intensities at the proposed 
geometries nevertheless survive at large $J$, a pronounced $r$ dependence of these rates is present. Similarly, it is possible to maximize the absolute value of any factor (34)-(37). For instance, $\left|G_{F}(Q R / R Q / Q P / P Q)\right|$ reaches the maximum $(2 / 15)$ at $\mathbf{e}_{1}=\mathbf{e}_{2}, \mathbf{e}_{3}=\mathbf{e}_{4}, \mathbf{e}_{1} \perp \mathbf{e}_{3}$. Since the intensity scales as $\left|G_{F}\right|^{2}$, such polarization adjustments appear to be useful for detection of weak RFWM signals.

\section{VELOCITY AVERAGING}

The definition (1) of $\chi^{(3)}$ implies averaging over velocities of the emitters. In doing so, we shall neglect the minor effects produced by the dependence of $\hat{\Gamma}$ on $\mathbf{v}$. In this case, the dependence of $\chi^{(3)}$ on $\mathbf{v}$ arises solely from the Doppler shifts entering the LS resolvents. Besides, we assume the factorized form of $\rho_{S}$ and, in line with the preceding RFWM studies, ${ }^{30-32,41,43}$ suppose the velocity relaxation rate $\omega_{c}$ to be small compared to the inhomogeneous Doppler broadening $\Delta \omega_{D}$. For instance, in an atmospheric $\mathrm{OH}$-containing flame ( $T \approx 1700 \mathrm{~K}$ ), an estimation based on the rigid sphere model gives $\omega_{c} / \Delta \omega_{D} \approx 0.1$ implying the predominance of the inhomogeneous Doppler broadening. Under these assumptions, we shall demonstrate that the velocity averaging differently affects particular $\chi^{(3)}$ terms and that the difference is magnified as the Doppler effect increasingly prevails over the collisional rovibronic relaxation. Although the latter situation is not typical for the atmospheric flames, it can be realized at low pressures or for rapidly moving dissociation products to be considered in a separate publication. The $\chi^{(3)}$ terms can be classified into two types: in case a the first event is an emission of photon 2 whereas in case $\mathbf{b}$ the initial event is an absorption of photon 1 or 3 . Using a shorthand notation of the relaxation parameters, the velocity integrand in case a reads

$$
\begin{aligned}
& \left(\Delta \Omega_{4}-k_{4} v+i \Gamma_{4}\right)^{-1}\left(-\Delta \Omega_{2}+k_{2} v+i \Gamma_{2}\right)^{-1}\left(\Delta \Omega_{n}-\Delta \Omega_{2}\right. \\
& \left.\quad+\left(k_{2}-k_{n}\right) v+i \Gamma_{n}\right)^{-1}
\end{aligned}
$$

whereas in case $\mathbf{b}$ it is

$$
\begin{aligned}
& \left(\Delta \Omega_{4}-k_{4} v+i \Gamma_{4}\right)^{-1}\left(\Delta \Omega_{n}-k_{n} v+i \Gamma_{n}\right)^{-1}\left(\Delta \Omega_{n}-\Delta \Omega_{2}\right. \\
& \left.\quad+\left(k_{2}-k_{n}\right) v+i \Gamma_{n}\right)^{-1}
\end{aligned}
$$

where $v$ is the velocity projection onto the (common) beam propagation axis and $n$ equals 1 or 3 . Matching the majority of practical cases, we assume hereafter that all laser frequencies belong to the visible or UV range. Besides, their differences will be supposed to lie in the IR range so that the Doppler term $\left(k_{2}-k_{n}\right) v$ in the last denominator is much smaller than the damping parameter $\Gamma_{n}$ and can be neglected. The integrals $S_{\mathbf{m}}(\mathbf{m}=\mathbf{a}, \mathbf{b})$ of (38) and (39) are given via the error function $w(u)$ of the complex argument ${ }^{44}$ lying in the upper half plane, but the explicit expressions depend on whether two poles of the integrands lie in the same half plane (case b) or in the opposite half planes (case a)

$$
\begin{gathered}
S_{\mathbf{a}}=-\frac{i \pi^{1 / 2}}{k_{4} k_{2} \bar{v}^{2}\left(u_{4}-u_{2}\right)}\left[w\left(u_{4}\right)+w\left(-u_{2}\right)\right], \\
S_{\mathbf{b}}=-\frac{i \pi^{1 / 2}}{k_{4} k_{n} \bar{v}^{2}\left(u_{4}-u_{n}\right)}\left[w\left(u_{4}\right)-w\left(u_{n}\right)\right] .
\end{gathered}
$$

Here, $u_{n}=\left(\Delta \Omega_{n}+i \Gamma_{n}\right) / \bar{v} k_{n}(n=1,3,4), u_{2}=\left(\Delta \Omega_{2}\right.$ $\left.-i \Gamma_{2}\right) / \bar{v} k_{n}$, and $\bar{v}=\sqrt{k T / 2 m}$ is the mean velocity of the emitter. Based on the known asymptotical behavior of $w(u)$, we conclude that in the fast rovibronic relaxation limit $(\varkappa \equiv$ $\left.\Gamma_{n} / k_{n} \bar{v} \gg 1\right) S_{\mathbf{a}}$ and $S_{\mathbf{b}}$ are nothing but the functions (38) and (39) in which the Doppler terms are completely neglected. When the Doppler effect dominates $(\varkappa \ll 1), S_{\mathbf{b}}$ becomes frequency independent $\left(S_{\mathbf{b}} \approx 2 / k_{4} k_{n} \bar{v}^{2}\right)$ while $S_{a}$ retains its resonance features,

$$
S_{\mathbf{a}} \approx \frac{2}{k_{4} k_{2} \bar{v}^{2}}\left[i+\frac{1}{u_{4}-u_{2}}\right]
$$

and dominates at the exact resonance: $\left|S_{\mathbf{b}} / S_{\mathbf{a}}\right| \sim\left(\Gamma_{4}\right.$ $\left.+\Gamma_{2}\right) / k \bar{v} \ll 1$. This ratio decreases as the temperature is raised and/or the gas pressure is lowered. Such cancellation can be rationalized by considering the behavior of both terms in the time domain where the b-terms show dampened oscillations with $v k_{n}$ and thus are gradually washed out by the velocity integration as the role of dampening is alleviated. The integration affects the a-terms at much less extent since they vary in time much slower and reveal a non-oscillatory behavior at resonance. Almost perfect conditions to observe the cancellation effect are realized for photolysis products formed at low pressure with high recoil velocities. However, in atmospheric flames, as will be shown in a subsequent paper, $\varkappa$ is comparable to unity and the roles of $\mathbf{a}$ and $\mathbf{b}$ amplitudes remain almost equally important.

\section{SUMMARY}

For gaseous media with randomly oriented molecular angular momenta, the line-space approach provides advantageously the tools to derive the terms of the third-order susceptibility which are due to triple resonances. Thanks to the exploited symmetry-adapted bases, the derivation is substantially facilitated and is easily extended to all possible schemes of resonantly enhanced four-wave mixing. Due to the strict account for collisional effects, the exact relaxation matrices are incorporated in the RFWM expressions. In doing so, not only the previous theoretical results are reproduced, but the collision-induced satellite resonances hitherto missing in the conventional theory are quantitatively interpreted as well. With an appropriately chosen configuration of the pumped and probed levels, the satellite intensity can be associated with a single channel of the rotational state-to-state collisional transfer whose cross correlation times and their tensor properties can be then determined by polarization-resolved measurements. With the proviso that scalar effects give major contributions to the rotational relaxation rates, simple expressions for the RFWM responses as functions of polarization geometry were derived in the limit of large angular momentum quantum numbers. Also, it is shown that the partial RFWM amplitudes corresponding to different photon-molecule interaction sequences are affected by velocity averaging in a dissimilar way. The effect shows up markedly when Doppler broadening is predominant. The line-space techniques show considerable promise to investigate the RFWM theory of ensembles with anisotropic momentum distributions. 


\section{ACKNOWLEDGMENTS}

This work is supported by the Science and Technology Cooperation Program Switzerland-Russia (GA 128546), the Swiss National Science Foundation $(\mathrm{NSF}(\mathrm{CH})$ ) (Grant No. 200020_146387/1) and by the Russian Foundation for Basic Research, Grant No. 11-03-00448a.

\section{APPENDIX A: BASICS OF LS APPROACH}

We outline the basics of the projection operator techniques ${ }^{1,3}$ needed to derive Eq. (2). Define the total Liouville space $\mathcal{L}$ as a collection of operators $(X, Y, \ldots)$ acting on the variables of the "molecule+bath" system with the metric

$$
\langle\langle X \mid Y\rangle\rangle=\operatorname{Tr} \rho_{T} X^{\dagger} Y .
$$

Next, a dynamical subspace $\mathcal{L}_{D} \subset \mathcal{L}$ is introduced whose vectors have the form of $\left.\left|A I_{B}\right\rangle\right\rangle$, where $A \in \mathcal{L}_{S}$ and $I_{B}$ is the identity operator acting on the bath states, i.e., $I_{B}=\sum_{\alpha}|\alpha\rangle\langle\alpha|$, where $|\alpha\rangle$ form an arbitrary set of the bath eigenfunctions. Evidently, the scalar product of two vectors belonging to $\mathcal{L}_{D}$ reduces to the LS expression (3). If $\left.\left|K_{\sigma}^{(r)}\right\rangle\right\rangle$ are the basis vectors (4) in $\mathcal{L}_{S}^{(r)}$, then the operator

$$
\left.\hat{P}=\sum_{K, r, \sigma}\left|K_{\sigma}^{(r)^{\prime}}\right\rangle\right\rangle\left\langle\left\langle K_{\sigma}^{(r) \prime}\right| .\right.
$$

$\left(\left|K_{\sigma}^{(r) \prime}\right\rangle=\left|I_{B} K_{\sigma}^{(r)}\right\rangle\right)$ projects $\mathcal{L}$ onto $\mathcal{L}_{D}$ whereas $\hat{Q}=1-\hat{P}$ is a projector onto the orthogonal complement $\mathcal{L}_{Q}$ to $\mathcal{L}_{D}$, so that $\mathcal{L}=\mathcal{L}_{D} \oplus \mathcal{L}_{Q}$. Since the frequency $\omega$ and $\hat{L}$ appear in $\chi^{(3)}(1)$ as the difference $\omega-\hat{L}+i 0$, it is possible to introduce a complex variable, $z=\omega+i 0$, lying in the upper half plane. Applying the matrix equation

$$
(A-B)^{-1}=A^{-1}+(A-B)^{-1} B A^{-1}
$$

to $\left.\left.\left.\left|K_{\sigma}^{(r) \prime}(z)\right\rangle\right\rangle=\left|(i z-i \hat{L})^{-1}\right| K_{\sigma}^{(r) \prime}\right\rangle\right\rangle$ (with $A=z-\hat{Q} \hat{L}$ and $\left.B=\hat{P} \hat{L}=\sum_{N, r_{1}, \sigma_{1}}\left|N_{\sigma_{1}}^{\left(r_{1}\right)^{\prime}}\right\rangle\right\rangle\left\langle\left\langle N_{\sigma_{1}}^{\left(r_{1}\right)^{\prime}}\right| \hat{L}\right)$ and using the identity

$$
(z-\hat{Q} \hat{L})^{-1}=z^{-1}\left[1+(z-\hat{Q} \hat{L})^{-1} \hat{Q} \hat{L}\right],
$$

we derive the equation

$$
\begin{aligned}
\left.\left|K_{\sigma}^{(r)^{\prime}}(z)\right\rangle\right\rangle= & \left.(i z)^{-1}\left[\left|K_{\sigma}^{(r)}\right\rangle\right\rangle+\left|F_{K}(r, \sigma ; z)\right\rangle\right\rangle+\sum_{N}\left(\delta_{K N} \omega_{K}\right. \\
& \left.\left.-\Delta \Omega_{N K}(r)+i \Gamma_{N K}^{\prime}(r, z)\right)\left|N_{\sigma}^{(r) \prime}(z)\right\rangle \mid\right] .
\end{aligned}
$$

Here, the frequency-independent matrix $\Delta \hat{\Omega}(r)$ is defined via $\left\langle\left\langle N_{\sigma_{1}}^{\left(r_{1}\right)}\left|\hat{L}_{1}\right| K_{\sigma}^{(r)^{\prime}}\right\rangle\right\rangle=\Delta \Omega_{N K}(r) \delta_{r_{1} r} \delta_{\sigma_{1} \sigma}$, and

$$
\begin{aligned}
\left.\left|F_{K}(r, \sigma ; z)\right\rangle\right\rangle & \left.\equiv(i z-i \hat{Q} \hat{L})^{-1} \hat{Q} \hat{L}\left|K_{\sigma}^{(r)}\right\rangle\right\rangle \\
& \left.=\int_{0}^{\infty} e^{i z t}\left|f_{K}(r, \sigma ; t)\right\rangle\right\rangle d t
\end{aligned}
$$

is the one-sided Fourier transform (FT) of the "random force" $\left.\left.{ }^{11}\left|f_{K}(r, \sigma ; t)\right\rangle\right\rangle \equiv e^{-i \hat{Q} \hat{L} \hat{Q}^{t}} \hat{Q} \hat{L}\left|K_{\sigma}^{(r) \prime}\right\rangle\right\rangle \in \mathcal{L}_{Q}$ associated with the LS transition $K_{\sigma}^{(r) \prime}$. This force is strictly orthogonal to $\left.\left|K_{\sigma}^{(r)}\right\rangle\right\rangle$ and develops in time by the action of the effective Liouvillian $\hat{Q} \hat{L} \hat{Q}$. The relaxation operator $\hat{\Gamma}^{\prime}(r, z)$ in (A5) is defined by the matrix elements

$$
\begin{aligned}
& \left\langle\left\langle N_{\sigma_{1}}^{\left(r_{1}\right)}\left|\hat{L}(z-\hat{Q} \hat{L})^{-1} \hat{Q} \hat{L}\right| K_{\sigma}^{(r) \prime}\right\rangle\right\rangle \\
& \quad \equiv\left\langle\left\langle N_{\sigma_{1}}^{\left(r_{1}\right)}\left|\hat{L} \hat{Q}(z-\hat{Q} \hat{L} \hat{Q})^{-1} \hat{Q} \hat{L}\right| K_{\sigma}^{(r) \prime}\right\rangle\right\rangle \\
& \quad=\Gamma_{N K}^{\prime}(r, z) \delta_{r_{1} r} \delta_{\sigma_{1} \sigma}
\end{aligned}
$$

obeying the selection rules (6). Since $\left.\hat{Q}\left(\hat{L}_{S}+\hat{L}_{B}\right)\left|K_{\sigma}^{(r) \prime}\right\rangle\right\rangle$ $=0$, one has $\left.\hat{Q} \hat{L}\left|K_{\sigma}^{(r)^{\prime}}\right\rangle\right\rangle=\hat{Q} \hat{L}_{1}\left|K_{\sigma}^{\left.(r)^{\prime}\right\rangle}\right\rangle$ which shows the random forces to be entirely due to the bath-molecule interactions. Moreover, Eq. (A7) shows $i \Gamma_{N K}^{\prime}(r, z)$ to be the FT of the time cross correlation function, $\left\langle\left\langle f_{N}\left(r, \sigma ; 0\left|f_{K}(r, \sigma ; t)\right\rangle\right\rangle\right.\right.$. It is also worth noting that, owing to Eq. (6), the action of $(z-\hat{L})^{-1}$ on $\left.\left|K_{\sigma}^{(r)^{\prime}}\right\rangle\right\rangle$ does not mix the tensor labels. Projecting both sides of (A5) onto $\mathcal{L}_{D}$ and $\mathcal{L}_{Q}$, one obtains two sets of linear equations, one for $\left.\left.\left|K_{\sigma}^{(r)}(z)\right\rangle\right\rangle_{D} \equiv \hat{P}\left|K_{\sigma}^{(r) \prime}(z)\right\rangle\right\rangle$ and another for $\left.\left.\left|K_{\sigma}^{(r) \prime}(z)\right\rangle\right\rangle_{Q} \equiv \hat{Q}\left|K_{\sigma}^{(r)}(z)\right\rangle\right\rangle$. Provided the resolvent matrix $\hat{R}(z) \equiv\left(z-\hat{L}_{S}+i \hat{\Gamma}(r, z)\right)^{-1}(\hat{\Gamma}(r, z)=i \Delta \hat{\Omega}$ $+\hat{\Gamma}(r, z))$ is nonsingular and using Eq. (5), the solutions of Eq. (A5) require the same matrix inversion. Once it is done,

$$
\begin{aligned}
\left.\left.\left.\mid K_{\sigma}^{(r) \prime}(z)\right)\right\rangle_{D}=\hat{R}\left|K_{\sigma}^{(r) \prime}\right\rangle\right\rangle & \left.=\sum_{N} R_{K N}\left|N_{\sigma}^{(r)}\right\rangle\right\rangle, \\
\left.\left.\left|K_{\sigma}^{(r) \prime}(z)\right\rangle\right\rangle_{Q}=\hat{R}\left|F_{K}(r, \sigma ; z)\right\rangle\right\rangle & \left.=\sum_{N} R_{K N}\left|F_{N}(r, \sigma ; z)\right\rangle\right\rangle .
\end{aligned}
$$

The rotations in $\mathcal{L}_{S}$ leave $\hat{L}_{S}, \Delta \hat{\Omega}$, and $\hat{\Gamma}^{\prime}$ invariant and, hence, transform $\left.\left|K_{\sigma}^{(r) \prime}(z)\right\rangle\right\rangle_{D}$ similar to the IST $\left.\left|K_{\sigma}^{(r) \prime}\right\rangle\right\rangle$; therefore, $\left.\left|K_{\sigma}^{(r)}(z)\right\rangle\right\rangle_{D}$ is an IST, too. However, the rotational irreducibility does not hold neither for the random forces nor for $\left.\left|K_{\sigma}^{(r) \prime}(z)\right\rangle\right\rangle_{Q}$, since the molecule-bath coupling $\hat{L}_{1}$ is affected by the LS rotations. What is more important, the projections (A8) and (A9) differently vary with the bath number density $n_{B}$ and their relative role follows from the dependences of $\left.\left|N_{\sigma}^{(r)}\right\rangle\right\rangle$ and $\left.\left.F_{N}(r, \sigma ; z)\right\rangle\right\rangle$ on $n_{B}$. When the S-B collisions are binary and uncorrelated, $F_{N}$ (as well as $\hat{\Gamma}$ ) scale linearly with $n_{B}$, in the same manner as $\hat{L}_{1}$ does, whereas the $\mathcal{L}_{D}$ basis vectors are invariant to the density. In this regime, the $\mathcal{L}_{Q}$ component (A9) scales versus $\left.\left|K_{\sigma}^{(r) \prime}(z)\right\rangle\right\rangle_{D}$ as a small parameter $n_{B} v_{0}$ (where $v_{0}$ is the volume occupied by an interacting pair) and thus can be neglected. Since $\left.\left|d_{4} I_{B}\right\rangle\right\rangle$ is a linear combination of the $\mathcal{L}_{D}$ basis vectors, one can substitute $\left.\left(\Omega_{a}^{\prime}+\Omega_{b}^{\prime}+\Omega_{c}^{\prime}-\hat{L}\right)^{-1}\left|d_{4} I_{B}\right\rangle\right\rangle$ in Eq. (1) by $\left.\hat{R}(z)\left|d_{4} I_{B}\right\rangle\right\rangle$ with $z=\Omega_{a}^{\prime}+\Omega_{b}^{\prime}+\Omega_{c}^{\prime}+i 0$. Considering the action of the next operator to the left (i.e., the molecule-field coupling $\hat{V}_{c}$ ) in (1), we notice that it transforms only the LS vectors so that $\hat{V}_{c} \hat{R}\left|d_{4} I_{B}\right\rangle \in \mathcal{L}_{D}$ holds. Evidently, the action of other propagators and molecule-field coupling operators can be processed in the same fashion. In doing so, we obtain the leading contribution to $\chi^{(3)}$ written as the LS scalar product (2).

\section{APPENDIX B: CALCULATION OF LS MATRIX ELEMENTS} have

Keeping in mind that $\hat{V}_{k}$ in (1) acts as a commutator, we

$$
\left\langle\left\langle I\left|V_{a}\right| K_{1}\right\rangle\right\rangle=-\rho_{m_{1} m_{1}}^{-1 / 2} \operatorname{Tr} \rho\left\{d_{a}\left(m_{1} n_{1}^{\times}\right)_{\sigma_{1}}^{(1)}-\left(m_{1} n_{1}^{\times}\right)_{\sigma_{1}}^{(1)} d_{a}\right\}
$$


in which the explicit form (4) of the transition operator $\left(m_{1} n_{1}^{\times}\right)_{\sigma_{1}}^{(1)}$ should be used. Besides, one has in the laboratory frame $d_{a}=\left(\mathbf{e}_{a}, \mathbf{d}\right)=\sum_{\mu}(-1)^{\mu} e_{a \mu} d_{-\mu}$. Next, the WignerEckart theorem ${ }^{29}$ is applied to the matrix element of $d_{-\mu}$ so that the reduced matrix element $\left\langle\alpha_{m_{1}} J_{m_{1}}\|d\| \alpha_{n_{1}} J_{n_{1}}\right\rangle$ $\equiv d_{m_{1} n_{1}}$ appears. The summation over the magnetic quantum numbers in (4) leads to the selection rule $\mu=\sigma_{1}$, and we obtain

$$
\begin{aligned}
\left\langle\left\langle I\left|V_{a}\right| K_{1 \sigma_{1}}^{(1)}\right\rangle\right\rangle= & -\left(3 \rho_{m_{1} m_{1}}\right)^{-1 / 2}\left[\rho_{m_{1} m_{1}}-\rho_{n_{1} n_{1}}\right] d_{m_{1} n_{1}} \\
& \times e_{a \sigma_{1}}(-1)^{J_{n_{1}}-J_{m_{1}}} .
\end{aligned}
$$

Next, we are to sum over $\sigma_{1}$,

$$
\begin{aligned}
\sum_{\sigma_{1}} & e_{a \sigma_{1}}\left\langle\left\langle K_{1^{\prime} \sigma_{1}}^{(1)}\left|V_{b}\right| K_{2 \sigma_{2}}^{\left(r_{2}\right)}\right\rangle\right\rangle \\
= & -\rho_{m_{1} m_{1}}^{-1 / 2} \rho_{m_{2} m_{2}}^{-1 / 2} \\
& \times \sum_{\sigma_{1} \mu M_{m_{1}^{\prime}} M_{n_{1}^{\prime}} M_{m_{2}} M_{n_{2}}} e_{a \sigma_{1}} e_{b \mu}(-1)^{\mu+J_{m_{1}^{\prime}}-M_{m_{1}^{\prime}}+J_{m_{2}}-M_{m_{2}}} \\
& \times C_{J_{n_{1}^{\prime}} M_{n_{1}^{\prime}} J_{m_{1}^{\prime}}-M_{m_{1}^{\prime}}} C_{J_{n_{2}} M_{n_{2}} J_{m_{2}}-M_{m_{2}}}^{r_{2} \sigma_{2}} \\
& \times \operatorname{Tr} \rho\left|\alpha_{m_{1}^{\prime}} J_{m_{1}^{\prime}} M_{m_{1}^{\prime}}\right\rangle\left\langle\alpha_{n_{1}^{\prime}} J_{n_{1}^{\prime}} M_{n_{1}^{\prime}}\right|\left[d_{-\mu},\left|\alpha_{n_{2}} J_{n_{2}} M_{n_{2}}\right\rangle\right. \\
& \left.\times\left\langle\alpha_{m_{2}} J_{m_{2}} M_{m_{2}}\right|\right],
\end{aligned}
$$

where [...] is the commutator. The trace is calculated in a similar manner,

$$
\begin{aligned}
\operatorname{Tr} \rho\left|\alpha_{m_{1}^{\prime}} J_{m_{1}^{\prime}} M_{m_{1}^{\prime}}\right\rangle\left\langle\alpha_{n_{1}^{\prime}} J_{n_{1}^{\prime}} M_{n_{1}^{\prime}}\right|\left[d_{-\mu},\left|\alpha_{n_{2}} J_{n_{2}} M_{n_{2}}\right\rangle\left\langle\alpha_{m_{2}} J_{m_{2}} M_{m_{2}}\right|\right] \\
=\rho_{m_{1}^{\prime} m_{1}^{\prime}}\left\{\delta_{m_{1}^{\prime} m_{2}} d_{n_{1}^{\prime} n_{2}}(-1)^{J_{n_{1}^{\prime}}-M_{n_{1}^{\prime}}}\left(\begin{array}{ccc}
J_{n_{1}^{\prime}} & 1 & J_{n_{2}} \\
-M_{n_{1}^{\prime}} & -\mu & M_{n_{2}}
\end{array}\right)\right. \\
\left.\quad-\delta_{n_{1}^{\prime} n_{2}} d_{m_{2} m_{1}^{\prime}}(-1)^{J_{m_{2}}-M_{m_{2}}}\left(\begin{array}{ccc}
J_{m_{2}} & 1 & J_{m_{1}^{\prime}} \\
-M_{m_{2}} & -\mu & M_{m_{1}^{\prime}}
\end{array}\right)\right\}, \text { (B4) }
\end{aligned}
$$

where $\delta_{k l}$ is a short-hand notation of $\delta_{J_{k} J_{l}} \delta_{\alpha_{k} \alpha_{l}} \delta_{M_{k} M_{l}}$. By using Eq. (12.1.3.6) of Ref. 29, the triple sum over $M_{m_{1}^{\prime}}, M_{n_{1}^{\prime}}$, and $M_{n_{2}}$ is reduced to the product of the $6 j$ - and $3 j m$-symbols, and we arrive at the following expression for the term proportional to $\delta_{m_{1}^{\prime} m_{2}}$ :

$$
\begin{aligned}
(-1)^{J_{n_{1}^{\prime}}-J_{m_{1}^{\prime}}} 3^{-1 / 2} \sum_{\sigma_{1} \mu M_{m_{1}^{\prime}} M_{n_{1}^{\prime}} M_{n_{2}}} e_{\alpha \sigma_{1}} e_{\beta \mu}(-1)^{\mu} \\
\quad \times C_{J_{n_{1}^{\prime}}^{\prime} M_{n_{1}^{\prime}} J_{m_{1}^{\prime}}-M_{m_{1}^{\prime}}} C_{J_{n_{2}} M_{n_{2}} J_{m_{1}^{\prime}} \sigma_{2}-M_{m_{1}^{\prime}}} \\
\times(-1)^{J_{n_{1}^{\prime}}-M_{n_{1}^{\prime}}}\left(\begin{array}{ccc}
J_{n_{1}^{\prime}} & 1 & J_{n_{2}} \\
-M_{n_{1}^{\prime}} & -\mu & M_{n_{2}}
\end{array}\right) \\
=T_{n_{2} n_{1}^{\prime} m_{2}}^{r_{2}}\left\{e_{a}^{(1)} \otimes e_{b}^{(1)}\right\}_{\sigma_{2}}^{\left(r_{2}\right)} .
\end{aligned}
$$

The same routine for the term containing $\delta_{n_{1}^{\prime} n_{2}}$ results in $-(-1)^{J_{n_{1}^{\prime}}-J_{m_{1}^{\prime}}}\left\{e_{b}^{(1)} \otimes e_{a}^{(1)}\right\}_{\sigma_{2}}^{\left(r_{2}\right)} T_{m_{2} m_{1}^{\prime} n_{2}}^{r_{2}}$. The last step is the summation over $\sigma_{3}$ of the product $\left\langle\left\langle K_{2^{\prime} \sigma_{2}}^{\left(r_{2}\right)}\left|V_{c}\right| K_{3 \sigma_{3}}^{(1)}\right\rangle\right\rangle\left\langle\left\langle K_{3^{\prime} \sigma_{3}}^{(1)} \mid\left(\mathbf{e}_{d}, \mathbf{d}\right)\right\rangle\right\rangle$ in which the second multiplier is easily found to be

$$
\left\langle\left\langle K_{3^{\prime} \sigma_{3}}^{(1)} \mid\left(\mathbf{e}_{4}, \mathbf{d}\right)\right\rangle\right\rangle=\rho_{m_{3}^{\prime} m_{3}^{\prime}}^{1 / 2} e_{4-\sigma_{3}}(-1)^{\sigma_{3}} d_{n_{3}^{\prime} m_{3}^{\prime}} / \sqrt{3}
$$

while the first one can be obtained by the same transformations as used to derive $\left\langle\left\langle K_{1 \sigma_{1}}^{(1)}\left|\widehat{V}_{b}\right| K_{2 \sigma_{2}}^{\left(r_{2}\right)}\right\rangle\right\rangle$. Finally, we have

$$
\begin{aligned}
\sum_{\sigma_{3}} & \left\langle\left\langle K_{2^{\prime} \sigma_{2}}^{\left(r_{2}\right)}\left|V_{c}\right| K_{3 \sigma_{3}}^{(1)}\right\rangle\right\rangle\left\langle\left\langle K_{3^{\prime} \sigma_{3}}^{(1)} \mid\left(\mathbf{e}_{4}, \mathbf{d}\right)\right\rangle\right\rangle \\
= & -\rho_{m_{2}^{\prime} m_{2}^{\prime}}^{1 / 2}(-1)^{J_{m_{2}^{\prime}}+J_{n_{2}^{\prime}}}(-1)^{\sigma_{2}} d_{n_{3}^{\prime} m_{3}^{\prime}} \\
& \times\left[\delta_{m_{2}^{\prime} m_{3}}\left\{e_{c}^{(1)} \otimes e_{4}^{(1)}\right\}_{-\sigma_{2}}^{\left(r_{2}\right)} T_{n_{3} n_{2}^{\prime} m_{3}}^{r_{2}} d_{n_{2}^{\prime} n_{3}}\right. \\
& \left.-\delta_{n_{2}^{\prime} m_{3}}\left\{e_{4}^{(1)} \otimes e_{c}^{(1)}\right\}_{-\sigma_{2}}^{\left(r_{2}\right)} T_{n_{3} m_{2}^{\prime} m_{3}}^{r_{2}} d_{m_{3} m_{2}^{\prime}}\right] .
\end{aligned}
$$

The summation over $\sigma_{2}$ results in Eq. (8).

\section{APPENDIX C: RECOUPLINGS IN GEOMETRICAL FACTORS}

To prove Eq. (30), we change the coupling order under the sign of the scalar product by applying Eq. (3.3.2.13) of Ref. 29 that results in

$$
G_{1423}(r)=(2 r+1) \sum_{g}(-1)^{g} T_{1 g 1}^{r} G_{1234}(g) .
$$

To proceed, the sum $W$ over $r$

$$
W=\sum_{r} \Pi_{r r}(-1)^{r} T_{1 g 1}^{r} T_{i f n}^{r} T_{i f n}^{r}
$$

is to be calculated which is done with the help of Eq. (12.2.3.18) of Ref. 29: $W=$ $(-1)^{-J_{i}-J_{n}-g-J_{f}-J_{f}} T_{f i f}^{g} T_{f n f}^{g}$. The phase factor is easily transformed by using the equality $(-1)^{-2 J}= \pm 1$ (in which the sign choice depends on whether the total electronic spin is integer or half integer), and we arrive at Eq. (30). Equation (31) is proved using the same procedure.

${ }^{1}$ D. Forster, Hydrodynamic Fluctuations, Broken Symmetry and Correlation Functions (Benjamin, Reading, MA, 1975).

${ }^{2}$ U. Fano, Phys. Rev. 131, 259 (1963).

${ }^{3}$ H. Mori, Prog. Theor. Phys. 33, 423 (1965).

${ }^{4}$ A. I. Burshtein and S. I. Temkin, Spectroscopy of Molecular Rotation in Gases and Liquids (Cambridge University Press, Cambridge, 1994).

${ }^{5}$ J.-M. Hartmann, C. Boulet, and D. Robert, Collisional Effects on Molecular Spectra (Elsevier, Amsterdam, 2008).

${ }^{6} \mathrm{~A}$. Levy, and N. Lacome, in The Spectroscopy of the Earth and Interstellar Medium, edited by C. Chackerian, K. N. Rao, Jr., and A. Weber, (Academic, New York, 1992), pp. 261-337.

${ }^{7}$ A. Ben-Reuven, Phys. Rev. 141, 34 (1966).

${ }^{8}$ N. N. Filippov and M. V. Tonkov, J. Chem. Phys. 108, 3608 (1998).

${ }^{9}$ A. P. Kouzov, Phys. Rev. A 60, 2931 (1999).

${ }^{10}$ P. Beaud, P. P. Radi, D. Franzke, H. M. Frey, B. Mischler, A. P. Tzannis, and T. Gerber, Appl. Opt. 37, 3354 (1998).

${ }^{11}$ G. Knopp, P. Radi, M. Tulej, T. Gerber, and P. Beaud, J. Chem. Phys. 118, 8223 (2003).

${ }^{12}$ V. Morozov, S. Mochalov, A. Kouzov, A. Olenin, and V. Tunkin, J. Raman Spectrosc. 34, 983 (2003).

${ }^{13}$ B. Lavorel, Ha Tran, E. Hertz, O. Faucher, P. Joubert, M. Motzkus, T. Buckup, T. Lang, H. Skenderovi, G. Knopp, P. Beaud, and H. M. Frey, C. R. Phys. 5, 215 (2004).

${ }^{14}$ P. J. Wrzesinski, H. U. Stauffer, W. D. Kulatilaka, J. R. Gord, and S. Roy, J. Raman Spectrosc. 44, 1344 (2013).

${ }^{15}$ J.-M. Hartmann, C. Boulet, T. Vieillard, F. Chaussard, F. Billard, O. Faucher, and B. Lavorel, J. Chem. Phys. 139, 024306 (2013).

${ }^{16}$ B. Lavorel, G. Millot, J. Bonamy, and D. Robert, Chem. Phys. 115, 69 (1987).

${ }^{17}$ A. Kouzov, D. Kozlov, and B. Hemmerling, Chem. Phys. 236, 15 (1998).

${ }^{18}$ T. Dreier, G. Schiff, and A. A. Suvernev, J. Chem. Phys. 100, 6275 (1994).

${ }^{19}$ A. Deroussiaux and B. Lavorel, J. Chem. Phys. 111, 1875 (1999). 
${ }^{20}$ B. Lavorel, G. Millot, R. Saint-Loup, H. Berger, L. Bonamy, J. Bonamy, and D. Robert, J. Chem. Phys. 93, 2176 (1990).

${ }^{21}$ Y. R. Shen, The Principles of Nonlinear Optics (Wiley, New York, 1984).

${ }^{22} \mathrm{~S}$. Mukamel, Principles of Nonlinear Optical Spectroscopy (Oxford University Press, Oxford, 1995).

${ }^{23}$ Actually, to create coherent responses, ns laser pulses are commonly used. Since the latter are much longer than the relaxation times, one may apply the stationary (frequency-domain) description.

${ }^{24}$ A. P. Kouzov and P. P. Radi, Phys. Rev. A 63, 010701 (2000).

${ }^{25}$ A. Kouzov and P. Radi, Proc. SPIE 4460, 144 (2002).

${ }^{26}$ X. Chen, B. D. Patterson, and T. B. Settersten, Chem. Phys. Lett. 388, 358 (2004).

${ }^{27}$ X. Chen and T. B. Settersten, Appl. Opt. 46, 3911 (2007).

${ }^{28}$ X. Chen, T. B. Settersten, and A. P. Kouzov, J. Raman Spectrosc. 40, 847 (2009).

${ }^{29}$ D. A. Varshalovich, A. N. Moskalev, and V. K. Khersonskii, Quantum Theory of Angular Momentum (World Scientific, Singapore, 1988), Chap. 3.

${ }^{30}$ S. Williams, R. N. Zare, and L. A. Rahn, J. Chem. Phys. 101, 1072 (1994).

${ }^{31}$ S. Williams, J. D. Tobiason, J. R. Dunlop, and E. A. Rohlfing, J. Chem. Phys. 102, 8342 (1995).
${ }^{32}$ S. Williams, E. A. Rohlfing, L. Rahn, and R. N. Zare, J. Chem. Phys. 106, 3090 (1997).

${ }^{33}$ T. Müller, T. A. W. Wasserman, P. H. Vaccaro, and B. R. Johnson, J. Chem. Phys. 108, 4 (1998).

${ }^{34}$ P. Maksyutenko, P. P. Radi, D. N. Kozlov, and A. P. Kouzov, J. Raman Spectrosc. 44, 1349 (2013).

${ }^{35}$ R. E. Teets, Opt. Lett. 9, 226 (1984).

${ }^{36}$ That is the $\chi^{(3)}$ tensor contracted with all unit polarization vectors involved.

${ }^{37}$ Y. Prior, IEEE J. Quantum Electron. QE-20, 37 (1984).

${ }^{38}$ M. Ducloy and D. Bloch, Phys. Rev. A 30, 3107 (1984).

${ }^{39}$ A. P. Kouzov and V. A. Krasheninnikov, Chem. Phys. 126, 301 (1988).

${ }^{40}$ X. Bruet, L. Bonamy, and J. Bonamy, Phys. Rev. A 62, 062702 (2000).

${ }^{41}$ S. Williams, L. A. Rahn, and R. N. Zare, J. Chem. Phys. 104, 3947 (1996).

${ }^{42}$ A. E. Bracamonte and P. Vaccaro, J. Chem. Phys. 119, 887 (2003).

${ }^{43}$ P. R. Berman, D. G. Steel, G. Khitrova, and J. Liu, Phys. Rev. A 38, 252 (1988).

${ }^{44}$ Handbook of Mathematical Functions with Formulas, Graphs, and Mathematical Tables, Applied Mathematical Series Vol. 55, edited by M. Abramovitz and I. A. Stegun (National Bureau of Standards, 1964), Chap. 7. 